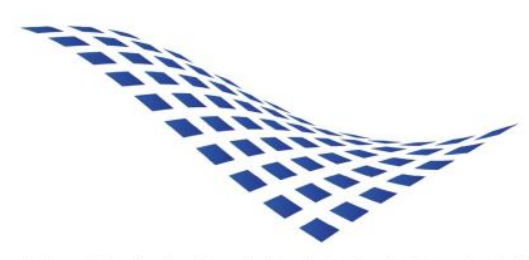

MOHAMMED VI

$P$ O L Y T E C H N I C EACULTÉ DE GOUVERNANCE,

$U N|V E R S I T Y| \begin{aligned} & \text { SCIENCES ECOALES } \\ & \text { SOMIQUES }\end{aligned}$

\title{
Coherent Diversification Measures in Portfolio Theory: An Axiomatic Foundation
}

CAHIER

DE RECHERCHE

21-02

Gilles Boevi KOUMOU Georges DIONNE 


\title{
Coherent Diversification Measures in Portfolio Theory: An Axiomatic Foundation *
}

\author{
Gilles Boevi KOUMOU ${ }^{+}$Georges DIONNE *
}

February 2, 2021

\begin{abstract}
We provide an axiomatic foundation for the measurement of correlation diversification measures in a one-period portfolio model. We propose a set of nine desirable axioms for this class of diversification measures. We name the measures satisfying these axioms coherent correlation diversification measures which we distinguish from coherent risk measures. We provide the decision theoretic foundations of our axioms by studying their compatibility with investors' preference for diversification in rank-dependent expected utility theory. We examine some of the most frequently used methods for measuring correlation diversification in terms of our axioms. Lastly, we explore whether our axioms have a representation function.
\end{abstract}

JEL Classifications: D81, G1, G11

Keywords: Portfolio Theory, Portfolio Diversification, Preference for Diversification,Correlation Diversification, Rank-Dependent Expected Utility Theory.

*Corresponding author: Gilles Boevi KOUMOU, Gilles.KOUMOU@um6p.ma

${ }^{\dagger}$ Facult e de gouvernance science 'economiques et sociales, Universit 'e Mohammed VI Polytechnique, Lot

66o, Hay Moulay Rachid, Ben Gu érir, 4315o Maroc. Email: Gilles.KOUMOU@um6p.ma

${ }^{\ddagger}$ Canada Research Chair in Risk Management, Department of Finance, HEC Montr 'eal, 30oo, chemin de la Côte-Sainte-Catherine, Montr'eal, Qu ébec, H3T 2A7, Canada. Email: georges.dionne@hec.ca. 


\section{Introduction}

Diversification is one of the major components of decision making in conditions of risk and uncertainty, especially in portfolio theory (see Markowitz, 1952; Ross, 1976; Sharpe, 1964). It consists of investing in various assets. Diversification is intended to reduce risk, particularly the likelihood and severity of portfolio loss, through multilateral insurance in which each asset is insured by the other assets. Despite criticism after the 2007-2009 financial crisis (see Holton, 2009), diversification is still an important risk management tool for many institutions and regulators. Its measurement and management, outside the standard risk measurement frameworks (e.g. the theory of monetary risk measures of Artzner et al (1999) and Föllmer and Weber (2015)), remain of fundamental importance in finance and insurance economics.

\section{I.I Existing Diversification Measures}

Following Markowitz's (1952) pioneering work on the mathematical formulation of diversification in portfolio theory, several measures of portfolio diversification have been proposed. According to Koumou (2019), there are four categories of diversification measures: law of large numbers diversification measures, correlation diversification measures, market portfolio diversification measures, and risk contribution diversification measures.

\section{I.I.I Law of Large Numbers Diversification Measures}

This category includes measures designed to capture the effect of law of large numbers diversification. This diversification strategy involves investing a small fraction of wealth in each of a large number of assets. A specific example is naive diversification (or equal weight portfolio), in which the same amount of wealth is invested in each available asset. Examples of law of large numbers (and in particular, naive diversification) measures are the effective number of constituents (see Carli, Deguest, and Martellini, 2014; Deguest, Martellini, and Meucci, 2013) and Bouchaud, Potters, and Aguilar's (1997) class of measures, which includes the Shannon and Gini-Simpson indexes (Zhou, Cai, and Tong, 2013). Other examples of naive diversification measures can be found in the studies by Yu, Lee, and Chiou (2014) and Lhabitant (2017). 


\section{I.I.2 Correlation Diversification Measures}

This category includes measures designed to capture the effect of correlation ${ }^{1}$ diversification. This diversification strategy is at the core of most decision theories, including the expected utility theory and Yaari's (1987) dual theory, and is related to the notion of correlation aversion (see Epstein and Tanny, 1980; Richard, 1975). It can be viewed as the rational diversification principle for risk-averse investors. It exploits interdependence between asset returns to reduce portfolio risk. The idea is that the less positively correlated the assets, the lower the likelihood they do poorly simultaneously in the same proportion, and the better the protection offered by diversification. Therefore, when there is correlation, it becomes sub-optimal to follow the law of large numbers diversification principle ex-ante. Correlation diversification is recommended in Basel II (CEBS, 2010), Basel III (BCBS, 2010, 2013), and in Solvency II for calculating the solvency capital requirement (CEIOPS, 2010 a,b). Examples of correlation diversification measures are Embrechts, Furrer, and Kaufmann's (2009) class of measures, Tasche's (2006) class of measures, the diversification ratio of Choueifaty and Coignard (2008), the diversification return of Booth and Fama (1992), the excess growth rate of Fernholz (2010), the return gap of Statman and Scheid (2005), Goetzmann and Kumar's (2008) measure of diversification, and the diversification delta of Vermorken, Medda, and Schroder (2012).

\section{I.I.3 Market Portfolio Diversification Measures}

This category includes measures designed to capture the effect of market portfolio diversification. This diversification strategy, introduced by Sharpe (1964), consists of holding a market portfolio or a market capitalization-weighted portfolio. It focuses on idiosyncratic risk reduction, so it may fail during systematic crashes like the 2007-2009 financial crisis. Examples of market portfolio diversification measures are portfolio size (see Evans and Archer, 1968), Sharpe's (1972) measure and Barnea and Logue’s (1973) measures.

\footnotetext{
${ }^{1}$ The term correlation here refers to any dependence measure including dissimilarity or similarity mea-sures.
} 


\section{I.I.4 Risk Contribution Diversification Measures}

The last category includes measures designed to capture the effect of risk contribution diversification. This diversification strategy, also known as risk parity, became popular after the 2007-2009 financial crisis (Maillard, Roncalli, and Teiletche, 2010; Qian, 2006). It consists of allocating portfolio risk equally among its components. Examples of risk contribution diversification measures are the effective number of correlated bets (see Carli, Deguest, and Martellini, 2014; Roncalli, 2014) and the effective number of uncorrelated bets of Meucci (2009) and Meucci, Santangelo, and Deguest (2014).

\section{I.2 Toward an Axiomatic foundation of Correlation Diversification Measures}

We focus on the rich choice set of correlation diversification measures. A completely rigorous axiomatic foundation for correlation diversification measurement is lacking in the literature. None of the existing measures truly has theoretical foundations (axiomatic or decision-theoretic foundations). Although correlation diversification is at the core of most decision theories and is recommended by international regulatory agencies, conceptual problems involved in its measurement have been overlooked. Much of the academic literature on the theoretical foundations of risk management has focused on the study of risk measurement (see Artzner et al, 1999; Föllmer and Schied, 2002; Frittelli and Gianin, 2002, 2005; Rockafellar, Uryasev, and Zabarankin, 2006). Even if correlation diversification is taken into account in the standard risk measurement frameworks through the properties of convexity, sub-additivity, comonotonic additivity, homogeneity and non-additivity for independence, these risk measures do not quantify the correlation diversification effect properly. The reason is that risk reduction is not equivalent to diversification. Diversification implies risk reduction, but the reverse is not true, because risk can also be reduced by concentration. For example, in Artzner et al's (1999) and Föllmer and Weber's (2015) monetary risk measurement theories, the possibility of reducing risks by concentration is taken into account through the property of monotonicity, and is as important as diversification. Consequently, standard risk measurement frameworks fail to adequately quantify and manage correlation diversification, except in the extreme case where all assets have the same risk. 
The lack of rigorous theories of correlation diversification measures when the decision maker is risk averse does not favor (i) rapid improvement in understanding the concept of diversification, (ii) development of coherent measures, and (iii) comparison of existing measures. The 2007-2009 financial crisis revealed that the concept of correlation diversification is misunderstood (Ilmanen and Kizer, 2012; Miccolis and Goodman, 2012; Statman, 2013). An example of the development of an inadequate correlation diversification measure is the diversification delta introduced by Vermorken, Medda, and Schroder (2012) and revised by Salazar Flores et al (2017).

Our paper is a first step toward a rigorous theory of correlation diversification measures. We provide an axiomatic foundation for the measurement of correlation diversification in a one-period portfolio model under the assumption that the investor has complete information about the joint distribution of asset returns.

Specifically, in Section 3, we present and discuss a set of minimum desirable axioms that a measure of portfolio diversification must satisfy in order to be considered coherent. In Section 4, we provide decision-theoretic foundations of our axioms by studying their rationality with respect to rank-dependent expected utility (RDEU) theory, one of the most promising non-expected utility theories in the literature. More specifically, we examine the compatibility of our axioms with investors' preference for diversification (PFD) in RDEU theory. We proceed in two steps. First, using the notion of PFD introduced by Dekel (1989) and extended by Chateauneuf and Tallon (2002) and Chateauneuf and Lakhnati (2007), we identify the measure of portfolio diversification at the core of RDEU theory as the differential between the weighted average of each asset risk and portfolio risk, similar to the study by Embrechts, Furrer, and Kaufmann (2009), where risk is measured by the standard certainty equivalent or risk premium. Next, we test the identified measure against our axioms. If the identified measure satisfies our axioms, we consider our axioms rationalized by RDEU theory. We distinguish three cases, and we show that

(i) if the utility function $u($.)is nonlinear and concave and the distortion function $h$ (.)js linear (in which case RDEU theory reduces to expected utility theory), then our axioms are rationalized by RDEU theory if and only if one of the following 
conditions is satisfied:

(a) risk is small in the sense of Pratt (1964) and absolute risk aversion is constant (see Proposition 1);

(b) each distribution of asset returns belongs to the location-scale family (including the elliptical distribution) and the certainty equivalent has a particular additive-separable form (see Proposition 2);

(ii) if the utility function is linear and the distortion function is nonlinear (in which case RDEU theory reduces to Yaari's (1987) dual theory) our axioms are rationalized by RDEU theory if and only if the distortion function is convex (see Proposition 3);

(iii) if the utility and the distortion functions are nonlinear and are continuously differentiable, then our axioms are rationalized by RDEU theory if and only if one of the following conditions is satisfied:

(a) risk is small in the sense of Pratt (1964) and the absolute risk aversion of the utility function $v(\cdot)^{2}$ is constant (see Proposition 4);

(b) each distribution of asset returns belongs to the location-scale family (including the elliptical distribution) and the certainty equivalent of $v($.)has a particular additive-separable form (see Proposition 5);

These results strengthen the economic desirability, reasonableness and relevance of our axioms.

In Section 5, we examine some of the most frequently used methods for measuring correlation diversification in terms of the axioms. This list includes:

(i) portfolio variance, a risk measure from the mean-variance model, often used to capture the benefit of diversification (Markowitz, 1952, 1959; Sharpe, 1964) and formally analyzed by Frahm and Wiechers (2013) as a measure of portfolio diversification;

(ii) the diversification ratio designed by Choueifaty and Coignard (2008), used by the firm TOBAM3 3 to manage billions in assets via its Anti- strategies in Benchmark $^{\text {B }}$

${ }^{2} v()=.u() \times h^{\prime}($.$) with h^{\prime}($.$) the derivative of the conjugate distortion function h^{-} .^{-}() \quad 1 \quad h$. 3https://www.tobam.fr/ 
Equities and Fixed Income. It coincides with the Sharpe ratio when each asset's volatility is proportional to its expected premium;

(iii) Embrechts, Furrer, and Kaufmann's (2009) class of measures and its normalized version analyzed by Tasche (2006), which are widely used to quantify diversification in both the finance and insurance industries (see Bignozzi et al, 2016; Dhaene, Denuit, and Vanduffel, 2009; Embrechts, Puccetti, and Rüschendorf, 2013; Embrechts, Wang, and Wang, 2015; Tong, Wu, and Xu, 2012; Wang, Bignozzi, and Tsanakas, 2015) and are recommended implicitly in some international regulatory frameworks (see BCBS, 2010; Bernard and Vanduffel, 2016).

We show that

(i) portfolio variance satisfies our axioms, under the very restrictive (if not impossible) conditions that assets have identical variances (see part (i) of Proposition 6);

(ii) the diversification ratio satisfies our axioms (see part (ii) of Proposition 6);

(iii) Embrechts, Furrer, and Kaufmann's (2009) (see part (iii) of Proposition 6) and Tasche's (2006) (see part (iv) of Proposition 6) classes of diversification measures satisfy our axioms, under the condition that the underlying risk measure is convex (or quasi-convex), homogeneous, translation invariant and reverse lower comonotonic additive.

These results

(i) strengthen our axioms. They are relevant to a number of measures with which we have considerable experience;

(ii) strengthen such measures as the diversification ratio, Embrechts, Furrer, and Kaufmann's (2009) and Tasche's (2006) classes of measures. Several desirability properties of these measures can be rationalized by the expected utility theory and Yaari's (1987) dual theory, and their popular use in empirical works and on the marketplace can be defended by our axioms;

(iii) reveal the limits of portfolio variance as an adequate measure of diversification in the mean-variance model. 
Since the diversification ratio coincides with the Sharpe ratio when each asset's volatility is proportional to its expected premium, the fulfillment of our axioms by the diversification ratio implies that the Sharpe ratio is a coherent correlation diversification measure under the above proportionality condition. It is also straightforward to verify that if the Sharpe ratio is a coherent correlation diversification measure, then each asset's volatility is proportional to its expected premium. As a result, the Sharpe ratio is a coherent correlation diversification measure if and only if each asset's volatility is proportional to its expected premium.

The conditions of fulfillment of our axioms by Embrechts, Furrer, and Kaufmann's (2009) and Tasche's (2006) classes of measures also establish the conditions under which a coherent risk measure in the sense of Artzner et al (1999) induces a coherent diversification measure. We show (Corollary 6) that a coherent risk measure induces a coherent diversification measure if and only if the coherent risk measure is reverse lower comonotonic additive. The Expected Shortfall (in the case of continuous distribution), which is chosen over the Value-at-Risk in Basel III (see BCBS, 2013), and the concave distortion risk measures (see Sereda et al, 2010) induce coherent diversification measures. These conditions also imply that a deviation risk measure (see Rockafellar, Uryasev, and Zabarankin, 2006) induces a coherent diversification measure, but the family of convex risk measures (see Föllmer and Schied, 2002; Frittelli and Gianin, 2002, 2005) does not. These conditions (part (iv) of Proposition 6 in particular) also support the findings of Salazar Flores et al (2017) that the diversification delta of Vermorken, Medda, and Schroder (2012) is an inadequate measure of portfolio diversification.

In Section 6, we investigate the structure of representation of our axioms. We show that our axioms imply a family of representations, but this family is not unique. We provide some examples and one counterexample of this family of representations to support our argument. Section 7 concludes the paper. Proofs are given in the appendix. Throughout the paper, vectors and matrices have bold style.

\section{I.3 Related Literature}

As mentioned above, the literature has focused mainly on the design of correlation diversification measures. Some studies have presented and discussed desirable axioms to 
support their proposed measures of diversification. For example, Choueifaty, Froidure and Reynier (2013) introduce the axiom of duplication invariance to support the diversification ratio. Evans and Archer (1968) present the monotonicity in portfolio size axiom to support portfolio size. The monotonicity in portfolio size axiom was also used by Rudin and Mor- gan (2006) to support the portfolio diversification index and by Vermorken, Medda, and Schroder (2012) to support the diversification delta. In addition to the axioms of duplication invariance and monotonicity in portfolio size, Carmichael, Koumou, and Moran (2015) discuss the axioms of degeneracy in portfolio size and degeneracy relative to dissimilarity to support Rao's Quadratic Entropy. Meucci, Santangelo, and Deguest (2015, Example 1) discuss the axiom of market homogeneity to support the effective number of bets. Our research contributes to this literature by generalizing, supplementing and rationalizing this list of axioms to obtain an axiomatic system for coherent correlation diversification measures.

In a recent contribution, De Giorgi and Mahmoud (2016 b) developed an axiomatic structure for a diversification measure that is designed to capture the effect of naive diversification. By contrast, our axiomatic system is relevant for measures based on the notion of correlation diversification, assuming that the risk-averse decision maker has complete information about the joint distribution of asset returns. Our work complements that of De Giorgi and Mahmoud (2016 b), but differs on an important point: their axiomatic system has a unique representative form and ours does not.

Finally, our work complements that of Artzner et al (1999) on risk measurement. Whereas Artzner et al (1999) provide a coherent axiomatic system of risk measures, our work provides a coherent axiomatic system of correlation diversification measures. Our work differs from that of Artzner et al (1999) in two other important respects. First, we study the rationality of our axiomatic system with respect to rank-dependent expected utility theory (Sections 4). Second, their axiomatic system implies a unique family of representations. 


\section{Preliminaries}

\section{I Notation}

We consider a one-period model, so time diversification is not analyzed. We assume that the investor is risk averse and the investment opportunity set is a universe $\mathrm{A}=\left\{A_{i}\right\}_{\bar{t} \mathbf{I}} \mathrm{N}$ of $N$ assets (risky or not), where $A_{i}$ denotes asset $i$ of $\mathrm{A} N \mathbb{1}=\{, N$ is fan index set and $N$ is a strictly positive integer $\left(N_{1}\right)$. We also assume that short sales are restricted and we denote by $\mathrm{W} \quad \mathbf{w}=w_{1}, \ldots,\left(w_{N}{ }^{\mathrm{T}} \mathrm{R}^{N}\right) \quad \mathrm{N}_{1} w_{i}: \mathbf{\Sigma}_{i}$ the set $\phi f$ long-only portfolios associated with , where $w_{i}$ As the weight of asset $i$ in portfolio $\mathbf{w}$, is a transpose operator and $\mathrm{R}$ is the set of positive real numbers. Our findings remain valid when short sales in the sense of Lintner (1965) are allowed, i.e. when the set of long/short portfolios is defined as $\mathrm{W}^{-} \quad \mathbf{w}$ $w_{1}, \ldots, \uplus_{N}^{\mathrm{TM}} \mathrm{T}=\mathbb{R}^{N} \quad N_{1} w_{)} \in \mathbf{1}$ wh $\mathrm{R}$ the=sef of real numbers and . the absolute |value

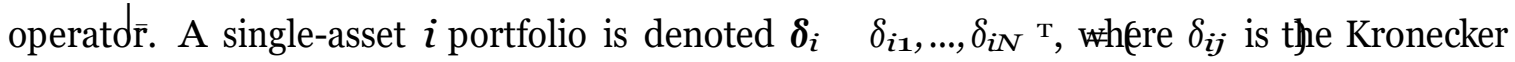
delta i.e. $\delta_{i i} \quad \mathbf{1}$ for each $i \quad N_{N}$ and $\delta_{i j} \quad 0$ førli $j, i, j \quad F_{N}$. A portfolio theld holds at least two assets is considered a diversified portfolio, while a portfolio that maximizes or minimizes a portfolio diversification measure is a well-diversified portfolio.

$R_{i} \in$ Rlenotes the future return of asset $i$, where IR $\Omega,, P$ isthe yector space of bounded real-valued random variables on a probability space $\Omega, P$, wherE $\Omega$ Is the set of states of nature, is the sigm\&-algebra of events, and $P$ is a sigma-additive probability measure on $\Omega$, We assu(metthat the investor has complete information about the joint distribution of $\mathbf{R}=\left(R_{1}, \ldots, R_{N}\right)^{\mathrm{T}}$. The expected value of $R_{i}$ is $\mu_{i}=\mathrm{E}\left(R_{i}\right)$, its variance $\sigma_{i}^{2}=\operatorname{Var}\left(R_{i}\right)$, its cumulative function $F_{R_{i}}\left(r_{i}\right)$, and its decumulative (survival) function $\bar{F}_{R_{i}}\left(r_{i}\right)=1-F_{R_{i}} r_{i}$, where $\mathrm{E}$. (a) Var . aije the operators of expectation and variance, respectively. The covariance between $R_{i}$ and $R_{j}$ is $\sigma_{i j}$ and the covariance matrix is $\mathbf{\Sigma}=$ $\left(\sigma_{i j}\right)_{i, j=1}^{N}$. The Pearson's correlation between $\boldsymbol{R}_{i}$ and $\boldsymbol{R}_{j}$ is $\rho_{i j}=\frac{\sigma_{i j}}{\sigma_{i} \sigma_{j}}$ and the correlation matrix is $\boldsymbol{\rho}=\left(\rho_{i j}\right)_{i, j=\mathbf{1}}^{N}$. The vector of asset volatility or standard deviation is denoted $\boldsymbol{\sigma}=\left(\sigma_{1}, \ldots, \sigma_{N}\right)^{\mathrm{T}}$. The future return of portfolio $\mathbf{w}$ is $R(\mathbf{w})=\mathbf{w}^{\mathrm{T}} \mathbf{R}$. Its expected value is $\mu(\mathbf{w})=\mathbf{w}^{\mathrm{T}} \boldsymbol{\mu}$ and its variance $\sigma^{2}(\mathbf{w})=\mathbf{w}^{\mathrm{T}} \boldsymbol{\Sigma} \mathbf{w}$. The cumulative and decumulative functions of $\mathbf{R}$ are $F_{\mathbf{R}}(\mathbf{r})$ and $F \mathbf{R}(\mathbf{r})$, respectively. When necessary, the subscript $i$ will be replaced by $A_{i}, \mathrm{~W}$ will be denoted $\mathrm{W}^{N}$ or $\mathrm{W}_{\mathrm{A}}^{N}$ and $\mathbf{w}$ will be denoted $\mathbf{w}_{\mathrm{A}}$. 


\subsection{Risk Measures}

In this section, we review some desirable properties of risk measures required for our analysis. For more details about these properties and for other desirable properties, readers are referred to the works of Pedersen and Satchell (1998), Artzner et al (1999), Song and Yan (2006), Song and Yan (2009), Sereda et al (2010), Föllmer and Schied (2010) and Wei, Ma, and $\mathrm{Hu}$ (2015).

Let $Q$ denote a risk measure o⿺R ${ }^{4}$ More formally, $Q$ is a mapping defined froı into $\mathrm{R}$, which satisfies a subset of the following set of desirable properties:

(a) Monotonicity: for all $X, Y \in \mathrm{R}$, if $X_{\leq} Y$, then $\left.Q_{(}{ }\right) \geq Q_{(} Y$;

(b) Sub-additivity: for all $X, Y \in \mathrm{R}, Q(X+Y) \leq Q(X)+Q(Y)$;

(c) Convexity: for all $X, Y \in \mathrm{R}, \lambda \in[0,1], Q(\lambda X+(1-\lambda) Y) \leq \lambda Q(X)+(1-\lambda) Q(Y)$

(d) Quasi-convexity: for all $X, Y \in \mathrm{R}, \lambda \in[0,1], Q(\lambda X+(1-\lambda) Y) \leq \max (Q(X) Q(Y))$;

(e) Comonotonic additivity: for comonotonic $X, Y \in \mathrm{R}, Q(X+Y)=Q(X)+Q(Y)$;

(f) Non-additivity for independence: for independent $X, Y \in \mathrm{R}, Q(X+Y) \neq Q(X)+$ $Q(Y)$

(g) Translation invariance: for all $a \in \mathrm{R}, X \in \mathrm{R}$ and $\eta \geq 0, Q(X+a)=Q(X)-\eta a$;

(h) Positive homogeneity: for all $\kappa \in \mathrm{R}, X \in \mathrm{R}$ and $b \geq 0, Q(b X)=b^{\kappa} Q(X)$;

(i) Law invariance: if $X, Y$ are identically distributed, denoted by $X=Y$, then $Q(X) \leq$ $Q(Y)$.

(j) Positivity: for all nonconstant $X, Q(X)>0$ and for all constant $X, Q(X)=0$.

(k) Reverse lower comonotonic additivity: for $X_{i} \in \mathrm{R}, i=1, \ldots, N, Q\left(\sum_{=1}^{N} X_{i}\right)=\sum_{=1}^{N} Q\left(X_{i}\right)$ implies that the sequence $X_{1}, \ldots, X_{N}$ is lower comonotonic.

A random vector $\mathbf{X}$ is comonotonic if and only if there are non-decreasing functions $f_{i}, i \in \mathrm{I}_{N}$ and a random variable $X$ such that $\mathbf{X} \stackrel{d}{=}\left(f_{1}(X), \ldots, f_{N}(X)\right)$, where $\stackrel{d}{=}$ stands for "equally distributed" (see Dhaene, Vanduffel, and Goovaerts, 2008). Intuitively, comonotonicity corresponds to an extreme form of positive dependency. All returns are driven positively, linearly or nonlinearly by a unique factor. For more details about the concept of comono-

${ }^{4}$ Note that $Q()=.\operatorname{Var}($.$) in the case of the variance risk measure.$ 
tonicity and its applications in finance, we refer readers to Dhaene et al (2002 a,b).

In a real-world environment, asset returns are usually not comonotonic, but can be comonotonic in the tails, as observed during the 2007-2009 financial crisis. The concept of upper comonotonicity was introduced and investigated by Cheung (2009). A random vector $\mathbf{X}$ is upper comonotonic if and only if $\mathbf{X}$ exhibits comotonicity behavior in the upper tail. Lower comonotonicity is the opposite: a random vector $\mathbf{X}$ is lower comonotonic if and only if its opposite $\mathbf{X}$ is upper comonotonic. We refer readers to Nam, Tang, and Yang (2011), Dong, Cheung, and Yang (2010) and Hua and Joe (2012) for more details on the concept of upper comonotonicity.

The property (a) is a natural requirement for a reasonable risk measure. The properties (b), (c), (d), (e) and (f) capture the diversification effect. The property (b) states that "a merger does not create extra risk" (Artzner et al, 1999). The properties (c) and (d) imply that diversification should not increase risk. The property of (e) implies that there is no benefit in terms of risk reduction to diversifying across comonotonic risks. The property (f) rules out the possibility that the pooling of independent risks does not have a diversification effect. The property (g) states that risk can be reduced by adding cash, except in the case where $\eta=0$. The property (i) states that a risk measure $Q(X)$ depends only on the distribution of $X$ i.e. $Q \times Q E_{X}$.(The property (h) states that a linear increase of the return by a positive factor leads to a non-linear increase in risk, except in the case where $\kappa 1$. The property (j) captures the idea that $Q$. measyres the degree of uncertainty in $X$. The property (k) requires that if $Q_{\left(\sum^{i}{ }_{1}^{N} X_{i}\right.}$ is additive, then risks $X_{1}, \ldots, X_{N}$ are lower comonotonic. In other words, if the benefit of diversification is exhausted, then risks are lower comonotonic.

Q(.) is called a coherent risk measure (see Artzner et al, 1999) if it satisfies (a), (b), (g) and (h) (with $\kappa=1), Q($.$) is called a convex risk measure (see Föllmer and Schied, 2002;$ Frittelli and Gianin, 2002, 2005), if it satisfies (a), (g) and (c). Q) is called a deviation risk measure (see Rockafellar, Uryasev, and Zabarankin, 2006), if it satisfies (b), (g) (with $\eta=0$ ), (h) (with $\kappa=1$ ) and (j). 


\subsection{Rank-Dependent Expected Utility Theory}

In this section, we review rank-dependent expected utility (RDEU) theory. We will use it to rationalize our axioms.

RDEU theory is one of the most promising non-expected utility theories in the literature. It is a generalized utility theory that embeds expected utility theory and Yaari's (1987) Dual Theory. It was first proposed by Quiggin (1982) under the name of anticipation utility theory and was further studied by many economists (see Abdellaoui, 2002; Chateauneuf and Wakker, 1999; Chew and Epstein, 1989; Chew, Epstein, and Wakker, 1993; Nakamura, 1995; Quiggin and Wakker, 1994; Segal, 1989, 1993 a,b; Wakker, 1994; Wakker and Tversky, 1993). It successfully explains many phenomena and paradoxes that are puzzling within the framework of expected utility theory, such as the Allais paradox, the simultaneous risk-averse and risk-seeking behavior, and the poor diversification and low stock market participation of households.

Preferences in RDEU theory are characterized by two elements: an outcome utility function, as in expected utility theory, and a probability weighting function, as in Yaari's (1987) dual utility Theory. More formally, assume thatchas RDEU theory representation. Then, from Denuit et al (2006) (see also Tsanakas and Desli, 2003),

$$
R_{i} \mathrm{c} R_{j} \Longleftrightarrow \mathrm{E}_{(u, h)}\left(w_{\mathbf{O}}\left(1+R_{i}\right)\right) \geq \mathrm{E}_{(u, h)}\left(w_{\mathbf{O}}\left(1+R_{j}\right)\right),
$$

where

$$
\left.\left.\mathbf{E}_{(u, \bar{h})}\left(w_{\mathbf{O}}(\mathbf{1}+R)\right)=\boldsymbol{J}_{-\infty}^{\mathrm{o}} \overline{(h} \overline{(F}_{u(u)(1+R))}(r)\right)-\mathbf{1}\right) d r+\boldsymbol{J}_{0}{ }^{\infty} \bar{h}\left(\bar{F}_{u\left(w_{(1+R)}\right.}(r)\right) d r,
$$

with $w_{\mathbf{o}}$ the investor's initial wealth, $u($.$) being a continuous, strictly increasing utility func-$ tion and unique up to positive affine transformations, and $h_{\uparrow}$ being a unique, continuous and strictly increasing distortion function from $[0,1]$ into $[0,1]$ satisfying $h(0)=-0$ and 
$\hbar(1)=1 . \mathrm{E}_{(u, h)}\left(w_{\mathrm{O}}(\mathbf{1}+R)\right)$ can also be rewritten as follows

$$
\mathrm{E}_{(u, \hbar)}\left(w_{\mathbf{O}}(\mathbf{1}+R)\right)=\mathbf{J}_{-\infty}^{\infty} u\left(w_{\mathbf{O}}(\mathbf{1}+r)\right) d h\left(F_{R}(r)\right)
$$

with $h($.$) being a conjugate distortion function of h($.$) such that h(x)=1-h(1-x)$.

If the support of the random variable $R$ is a finite set, $\mathrm{E}_{(u, h)}\left(w_{\mathbf{O}}(\mathbf{1}+R)\right)$ can be written as

$$
\mathrm{E}_{(u, \hbar)}\left(w_{\mathbf{O}}(\mathbf{1}+R)\right)=u\left(w_{\mathbf{O}}\left(\mathbf{1}+\boldsymbol{r}_{\mathbf{1}}\right)\right)+\sum_{i=2}^{T}\left(u\left(w_{\mathbf{O}}\left(\mathbf{1}+\boldsymbol{r}_{\boldsymbol{i}}\right)\right)-u\left(w_{\mathbf{O}}\left(\mathbf{1}+\boldsymbol{r}_{\mathbf{i - 1}}\right)\right)\right) h\left(\bar{v}_{i-\mathbf{1}}\right)
$$

with $v_{i}=\sum_{j i+1}^{T} p_{j}=P\left(R>r_{i}\right)$.

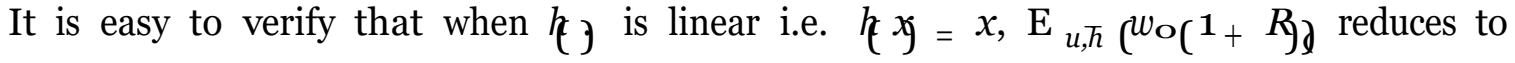

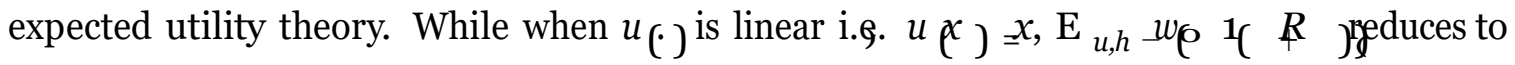
Yaari's (1987) dual utility function. In the rest of the paper, we assume that $w_{\mathbf{O}} \mathbf{1}$.

The investor's risk profile in RDEU theory is characterized by some conditions on $u_{\text {. }}$ and $\bar{h}$ (. )or $h$.(.)In this paper, we recall only the conditions about investor risk aversion:

(i) the investor with differentiable functions (u). and $\bar{h}($ ) (or equivalently $h($.$) ) is$ weakly risk averse (i.e. risk averse in the sense of $\mathrm{E}_{h} \not \mathrm{E}_{h} \mathrm{E}(R(\mathrm{E}) R=$ ) if( )

(a) $\exists k \geq 1$ such that $u^{\prime}(x) \leq k \frac{u(x)-u(y)}{x-y}, x<y$; and

(b) $h_{(x)} \leq x, \forall x \in[0,1]$ (or equivalently $h_{(x) \geq x, \forall}{ }^{x} \in[0,1]$ ).

(ii) the investor is strongly risk averse (i.e. risk averse in the sense of mean preserving spread as defined by De Giorgi and Mahmoud (2016 a, Definition 9, pp. 152)) if and only if $u($.$) is concave and \hbar($.$) is convex and \hbar(x) \neq x$ (or equivalently $h($.$) is$ concave and $h(x) \neq x)$.

In particular,

(i) the investor whose $u($ ) is concave is weakly risk averse if and only if he/she is weakly pessimistic under risk i.e. $\left\{\begin{array}{l}- \\ x\end{array}\right.$ or $h x(x) \geq 0,1 \forall . \in[\quad]$

(ii) the investor whose $u$ ( )is concave and satisfies for $\left.x \quad \varrho_{\bar{q}} \mathbb{1}, u\right] x \quad(x)^{2}, \nexists \quad 1$ is weakly risk averse if and only if $h(x) \leq x^{\bar{n}}$ or $h(x) \geq 1-(1-x)^{n}, \forall x \in[0,1]$. 
For more details on risk attitude in RDEU theory, we refer readers to Cohen (1995) and Ryan (2006).

\section{Axioms}

We now present and discuss a set of minimum desirable axioms that a measure of portfolio diversification must satisfy in order to be considered coherent. The next section provides the decision-theoretic foundations for these axioms. Let us first introduce the definition of a portfolio diversification measure.

How can we define a diversification measure of a portfolio w? Although there is no unique definition of diversification in portfolio theory, it is recognized that the diversification interest variable (the distribution of portfolio weight $\mathbf{w}$ ) and benefit (the reduction of risk or uncertainty) are unique. Let $\Phi$ be a continuous measure of portfolio diversification. In the case where the investor is risk averse and has complete information about the joint distribution of asset returns $\mathbf{R}$, it is natural to represent $\Phi$ as a mapping from $\mathrm{W}$ into $\mathrm{R}$ assigning to each $\mathbf{w}$ a real value $\Phi(\mathbf{w} \mathbf{R} \in \mathbf{R}$, with $\Phi, \mathbf{R}$ representing the degree of diversification of $\mathbf{w}$ conditional to $\mathbf{R}$ explicitly or implicitly. The form of the function $\Phi(. \mid \mathbf{R})$ depends on some properties of the portfolio diversification measure.

In the case where the investor has no information about $\mathbf{R}, \Phi$ can also be represented as a function of $\mathbf{w}$ i.e. $\Phi(\mathbf{w})$, and be used to capture the effect of naive diversification.

Let us now introduce our set of desirable axioms. There is no loss of generality in assuming that the well-diversified portfolio of $\Phi(\mathbf{w} \mid \mathbf{R})$, denoted $\mathbf{w}^{*}$, is obtained by maximization. Therefore, given a measure $\Phi$, we say that "portfolio $\mathbf{w}_{1}$ is more diversified than portfolio $\mathbf{w}_{2}{ }$ " if and only if $\Phi \mathbf{w}_{1} \mathbf{R} \geq \Phi\left(\mathbf{w}_{2} \mid \mathbf{R}\right)$.

Our first axiom formalizes investors' preference for diversification over W. This axiom was first formulated in Carmichael, Koumou, and Moran (2015) and is expressed in

Concavity (C). For each $\mathbf{w}_{1}$ and $\mathbf{w}_{2} \in \mathrm{W}, \alpha \in[0,1]$ and $\mathbf{R} \in \mathrm{R}^{N}$,

$$
\Phi\left(\alpha \mathbf{w}_{\mathbf{1}}+(\mathbf{1}-\alpha) \mathbf{w}_{\mathbf{2}} \mid \mathbf{R}\right) \geq \alpha \Phi\left(\mathbf{w}_{\mathbf{1}} \mid \mathbf{R}\right)+(\mathbf{1}-\alpha) \Phi\left(\mathbf{w}_{\mathbf{2}} \mid \mathbf{R}\right)
$$


with strict inequality for at least one $\alpha$.

Concavity implies that holding different assets increases total diversification. It also ensures that the diversification is always beneficial and can be decomposed across asset classes. Concavity can be replaced by a less restrictive axiom.

QUASI-CONCAVITY (QC). For each $\mathbf{w}_{1}$ and $\mathbf{w}_{2} \in \mathrm{W}, \alpha \in[0,1]$ and $\mathbf{R} \in \mathrm{R}^{N}$,

$$
\Phi\left(\alpha \mathbf{w}_{\mathbf{1}}+(1-\alpha) \mathbf{w}_{\mathbf{2}} \mid \mathbf{R}\right) \geq \min \left(\Phi\left(\mathbf{w}_{\mathbf{1}} \mid \mathbf{R}\right), \Phi\left(\mathbf{w}_{\mathbf{2}} \mid \mathbf{R}\right)\right)
$$

with strict inequality for at least one $\alpha$.

Our next axiom is complementary to Concavity. It is favored by Carmichael, Koumou, and Moran (2015) and is expressed in

Size Degeneracy (SD). There is a constant (for a normalization) $\Phi$ R such that for each $\mathbf{R} \in \mathrm{R}^{N}$,

$$
\Phi\left(\boldsymbol{\delta}_{i} \mid \mathbf{R}\right)=\underline{\Phi} \text { for each } i \in \mathbf{I}_{N} .
$$

Size Degeneracy imposes that all single-asset portfolios are equally desirable in terms of diversification. Size Degeneracy together with Concavity imply that diversification is always better than full concentration or specialization; formally for each $\mathbf{R} \in \mathrm{R}^{N}$ and for each $\mathbf{w} \in \mathrm{W}$

$$
\Phi\left(\boldsymbol{\delta}_{i} \mid \mathbf{R}\right) \leq \Phi(\mathbf{w} \mid \mathbf{R}) \text { for each } i \in \mathbf{I}_{N} .
$$

Size Degeneracy therefore strengthens investors' preference for diversification materialized by Concavity. It guarantees that $\mathbf{w}^{*}$, the well-diversified portfolio of $\Phi(\mathbf{w} \mid \mathbf{R})$, is a diversified portfolio when $\Phi(\mathbf{w} \mid \mathbf{R})$ satisfies Concavity. Clearly, the two axioms are necessary to prevent portfolio concentration on one asset from going undetected.

Our next axiom formalizes the behavior of $\Phi(\mathbf{w} \mid \mathbf{R})$ when $\mathbf{R}$ is homogeneous in the sense of perfect similarity. It is expressed in 
RIsk DEgEnERACY (RD). LetA $=\left\{A_{i}\right\}_{i}{ }_{N}$ be a universe of $N$ assets such that $\boldsymbol{A}_{i}$ e $A$, for each $i \in \mathrm{I}_{N}$. Then, for each $\mathbf{w} \in \mathrm{W}$

$$
\Phi(\mathbf{w} \mid \mathbf{R})=\underline{\Phi} .
$$

Risk Degeneracy ensures that there is no benefit to diversifying across perfectly similar assets. Such diversification is equivalent to full concentration. Risk Degeneracy is also necessary to keep portfolio concentration from going undetected. Risk Degeneracy generalizes Carmichael, Koumou, and Moran's (2015, Property 2) axiom of degeneracy relative to dissimilarity, which states that "a portfolio formed solely with perfectly similar assets must have the lowest diversification degree."

Our next axiom is complementary to Risk Degeneracy and is expressed in

REVERSE Risk DEgeneracy (RRD). Consider the relation $\Phi \mid \mathbf{w}) \mathbf{R} \Phi$ on $\mathrm{R}$ for each $\mathbf{w} \in \mathrm{W}$ such that $\mathbf{w} \neq \boldsymbol{\delta}_{i}, i \in \mathbf{I}_{N}$ and without loss of generality assume that $w_{i}>\mathbf{o}$, for each $i \in$ . Assume that a solution exists and is denoted as $\mathbf{R}^{*}$. Then $\mathbf{R}^{*}$ must be lower comonotonic. Note that $\mathbf{R}^{*}$ can be different from $\mathbf{R}$.

Reverse Risk Degeneracy is also necessary to prevent undetected portfolio concentration. It states that when $\Phi(\mathbf{w} \mid \mathbf{R})=\underline{\Phi}$ with $\mathbf{w}$ a diversified portfolio, $\mathbf{R}=\left(R_{i}\right]_{i}$ or its transformation is necessarily lower comonotonic, where $\mathbf{I}=\left\{i \mid w_{i}>0\right\}_{+}^{\in \mathbf{I}}$ The following example is provided to get a better sense of the importance of Reverse Risk Degeneracy.

Example 1 (Embrechts, FurRer, and Kaufmann's (2009) Class of Measures). Consider Embrechts, Furrer, and Kaufmann's (2009) class of diversification measures (see diversification measure (iii) in Section 5) when the risk measure $Q$.(i additive for independence i.e for independent $X, Y \in \mathrm{R}, Q(X+Y)=Q(X)+Q(Y)$. This is the case when $Q$ (is the mixed Esscher premium or the mixed exponential premium analyzed in Goovaertset al (2004). In that case, according to Embrechts, Furrer, and Kaufmann's (2009) classof diversification measures, any portfolio with independent asset returns and single-asset portfolios would have the same degree of diversification, which is counterintuitive. Reverse 
Risk Degeneracy rules out this sub-class of Embrechts, Furrer, and Kaufmann's (2009) and Tasche's (2006) diversification measures.

Our next axiom is the formalization of the property of duplication invariance of Choueifaty, Froidure and Reynier (2013) analyzed in Carmichael, Koumou, and Moran (2015). It is expressed in

DUPLICATION INVARIANCE (DI). LAł $=\left\{^{+}\right\}_{\in \mathbf{I}} \underset{+}{A_{i}^{+} i_{N}}$ be a universe of assets such that $A_{i}^{+}=A_{i}$, for each $i \in \mathbf{I}_{N}$ and $A_{N+1}^{+}=A_{k}$, for $k \in \mathbf{I}_{N}$. Then

$$
\begin{aligned}
\Phi\left(\mathbf{w}_{\mathrm{A}}^{*} \mid \mathbf{R}_{\mathrm{A}}\right) & =\Phi\left(\mathbf{w}_{\mathrm{A}^{+}}^{*} \mid \mathbf{R}_{\mathrm{A}^{+}}\right) \\
\mathbf{w}_{A_{i}}^{*} & =\mathbf{w}_{A_{i}^{*}}^{*} \text { for each } i \neq k, i \in \mathbf{I}_{N} \\
\mathbf{w}_{A_{k}}^{*} & =\mathbf{w}_{A_{k}^{*}}^{*}+\mathbf{w}_{A_{N^{*}+1}^{*}}^{*} .
\end{aligned}
$$

The reasonableness and relevance of Duplication Invariance is evident. It allows us to avoid risk concentration by ensuring that the optimal diversified portfolio is not biased toward multiple representative assets. It is necessary to prevent portfolio concentration from going undetected. The following example is provided to demonstrate the importance of Duplication Invariance.

EXAMPLE 2 (CASE $N$ 2). Consider the case where $N=2$. In that cas $₫ A=\left\{A_{1}, A_{2}\right\}$ and $\mathrm{A}^{+}=\left\{A_{1}, A_{2}, A_{3}^{+}\right\}$such that $A_{3}^{+}=A_{1}$. Duplication Invariance states that $(i)$ the optimal degree of diversification of $A_{A}$ and $\quad$ + must be equal; (ii) the optimal weight of $A_{2}$ in must be equal to the sum of the optimal weights of $A_{2}$ and $A_{3}^{+}$in $\mathrm{A}^{+}$.

Our next axiom formalizes the relationship between diversification and portfolio size. It is expressed in

Size Monotonicity (M). LeA ${ }^{++}=\left\{A_{i}^{++}\right\}_{i \in \mathbf{N}_{4}}$ be a universe of assets such that $A_{i}^{+^{+}}=$ $A_{i}$, for each $i \in \mathbf{I}_{N}$ and $A_{N+1}^{++} \neq A_{i}$, for each $i \in \mathbf{I}_{N}$. Then

$$
\Phi\left(\mathbf{w}_{\mathrm{A}^{+}}^{*} \mid \mathbf{R}_{\mathrm{A}^{++}}\right) \geq \Phi\left(\mathbf{w}_{\mathrm{A}}^{*} \mid \mathbf{R}_{\mathrm{A}}\right) .
$$


Size Monotonicity is natural in the portfolio diversification literature (see Carmichael, Koumou, and Moran, 2015; Evans and Archer, 1968; Rudin and Morgan, 2006; Vermorken, Medda, and Schroder, 2012). It reveals that portfolio diversification is a non-decreasing function of portfolio size.

Our next axiom is an adaptation of the translation invariance axiom of risk measures. It is expressed in

TRANSLATION INVARIANCE (TI). LetA $+a=\left\{\begin{array}{ll}\boldsymbol{A}_{i} & \boldsymbol{\theta}\end{array} \boldsymbol{\}}_{\Lambda \in}\right.$ be a universe of assets such that $R_{A_{i}+a}=R_{A_{i}}+a$, for each $i \in \mathrm{I}_{N}, a \in \mathrm{R}$. Then for each $\mathbf{w} \in \mathrm{W}_{\mathrm{A}}=\mathrm{W}_{\mathrm{A}+a}$,

$$
\Phi\left(\mathbf{w} \mid \mathbf{R}_{\mathrm{A}+a}\right)=\Phi\left(\mathbf{w} \mid \mathbf{R}_{\mathrm{A}}\right)
$$

The desirability of Translation Invariance comes from the translation invariance risk measure axiom. It implies that adding the same amount of cash to asset returns does not change the degree of portfolio diversification. Consider the translation invariance risk measure axiom. Assume that $\eta=0$. In that case, adding the same amount of cash to asset returns does not affect the degree of portfolio risk. The degree of portfolio diversification is not affected either. Now, assume that $\eta$ ○. In that case, adding the same amount of cash to asset returns reduces portfolio risk, but does not affect the degree of portfolio diversification.

However, when risk is defined as a capital requirement or a probability of loss (for example Expected Shortfall or Conditional Value-at-Risk), Translation Invariance can be seen as counterintuitive. As an illustration, consider the case where risk $Q$ ) is defined as a capital requirement verifying the property of translation invariance. Assume that $a \frac{e\left(w^{t} \mathbf{R}\right)}{\eta}$ with $\eta \neq 0$. Then $Q\left(\mathbf{w}^{\mathrm{T}} \mathbf{R} \quad \boldsymbol{t}\right) \boldsymbol{\theta}$, but $\Phi \mathbf{w}(\mathbf{R} \mid \quad$ a $\Phi=\mathbf{w}(\mathbf{R} \mid \quad \phi \lambda$ This counterintuitive result can be viewed as over diversification, ${ }^{\mathrm{A}}$ and can be interpreted as an extreme precaution against extreme risk.

In the case where $\Phi(, \mathbf{R})$ is a normalized measure, i.e. when $\Phi(\mathbf{w} \mid \mathbf{R})$ can be rewritten as follows

$$
\Phi(\mathbf{w} \mid \mathbf{R})=\frac{\tilde{\Phi} \mid \mathbf{R})}{Q\left(\mathbf{w}^{\top} \mathbf{R}^{\top}\right)}
$$


or equivalently

$$
\Phi(\mathbf{w} \mid \mathbf{R})=\frac{\tilde{\omega} \mid \mathbf{R})-Q\left(\mathbf{w}^{\mathrm{T}} \mathbf{R}\right)}{Q(\mathbf{w} \mathbf{R})}
$$

with $\Phi . \mathbf{R}$ a portfolio diversification measure such that $\tilde{\Phi} \mathbf{w} \mathbf{R} a \quad \tilde{\Phi} \mathbf{y} \mathbf{R}$, Translation Invariance must be replaced by the following axiom.

TRANSLATION INVARIANCE-2 (TI2). LAt $+a=\left\{A_{i}+a\right\}_{i}$ be a universe of assets such that $R_{A_{i}+a}=R_{A_{i}}+a$, for each $i \in \mathrm{I}_{N}$ with $a \in \mathrm{R}$. Then for each $\mathbf{w} \in \mathrm{W}_{\mathrm{A}+a}$

$$
\begin{aligned}
& \frac{\partial \Phi\left(\mathbf{w} \mid \mathbf{R}_{\mathrm{A}+a}\right)}{\partial a} \geq \mathbf{0}, \\
& { }_{a} \lim _{-\infty} \Phi\left(\mathbf{w} \mid \mathbf{R}_{\mathrm{A}+a}\right)=\underline{\underline{\Phi}}, \\
& \lim _{+\infty} \Phi\left(\mathbf{w} \mid \mathbf{R}_{\mathrm{A}+a}\right)=\Phi,
\end{aligned}
$$

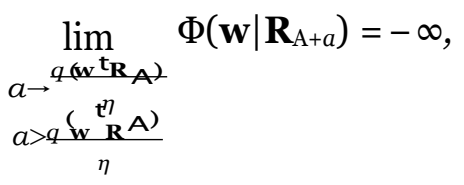

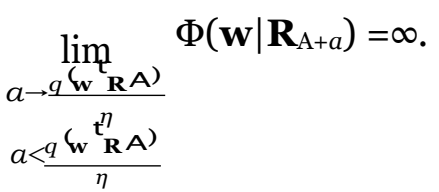

The idea behind Translation Invariance-2 is as follows. Equation (17) states that adding cash increases the diversification benefit. This is because adding cash reduces the total risk $Q($.$) and does not affect diversification \Phi$. $\mathbb{R} /$.)Equations (18) and (19) ensure that when cash converges to or , theodiversification benefit vanishes because the whole system becomes homogeneous. Equations (20) and (21) capture the overdiversification behavior of $\Phi$. R wḥфn fisk converges to o and diversification becomes unnecessary.

Our next axiom is an adaptation of the positive homogeneity axiom of risk measures. It is expressed in

Homogeneity (H). Let $b \mathrm{~A}=\left\langle 2 A_{i} \quad\right\}_{{ }_{\mathrm{Al}}}$ be a universe of assets such that $R_{b A_{i}}=b R_{A_{i}}$, for each $i \in \mathrm{I}_{N}$ with $b \geq 0$. Then there exists $\kappa \in \mathrm{R}$ such that for each $\mathbf{w} \in \mathrm{W}_{\mathrm{A}}=\mathrm{W}_{b \mathrm{~A}}$

$$
\Phi\left(\mathbf{w} \mid \mathbf{R}_{b \mathrm{~A}}\right)=b^{\kappa} \Phi\left(\mathbf{w} \mid \mathbf{R}_{\mathrm{A}}\right) .
$$


The desirability of Homogeneity comes naturally from the homogeneous property of the risk measure. It requires that $\Phi\left(\mathbf{w} \mid \mathbf{R}_{b A}\right)$ depend on the scalability of $\mathbf{R}$. This dependence is linear when $\kappa 1$ and nonlinear when $\kappa 1$ and $\neq \kappa$. In the case where $\Phi . \mathbf{R}$ is a nofmglized measure, $\kappa$ must be equal to zero, which ensures that $\Phi . \mathbf{R}$ (must not depend on scalability.

Our last axiom presents the behavior of $\Phi \mathbf{y} \mathbf{R}$ when $R_{1}, \ldots, R_{N}$ are exchangeable random variables. ${ }^{5}$ It is expressed in

Symmetry (S). If $R_{1}, \ldots, R_{N}$ are exchangeable, then $\Phi(\mathbf{w} \mid \mathbf{R})$ is symmetric in $\mathbf{w}$.

Symmetry states that a portfolio diversification measure must be symmetric in $\mathbf{w}$ if $R_{1}, \ldots, R_{N}$ are exchangeable. The idea behind Symmetry is that the exchangeable random variables imply homogeneous risks. Thus, the decision maker must be indifferent in terms of diversification between $\mathbf{w}$ and $\Pi \mathbf{w}$, where $\boldsymbol{\Pi}$ is a permutation matrix.

From Marshall, Olkin, and Arnold (2011, C.2. and C.3. Propositions, pp 97-98), Symmetry and Concavity or Quasi-Concavity taken together imply that $\Phi(\mathbf{w} \mathbf{R})$ is Schur-concave in $\mathbf{w}$ when $R_{1}, \ldots, R_{N}$ are exchangeable. As a result, $\Phi(\mathbf{w} \mid \mathbf{R})$ must be a measure of naive diversification and the optimal diversified portfolio $\mathbf{W}^{*}$ must be the naive portfolio $-1{ }_{N}$ when $R_{1}, \ldots, R_{N}$ are exchangeable. This result is consistent with the principle that the exchangeability assumption on $R_{1}, \ldots, R_{N}$ is equivalent to the assumption that the decision maker has no information about asset risk characteristics R. Moreover, Symmetry and Concavity or Quasi-Concavity taken together imply the axiom of market homogeneity analyzed in Meucci, Santangelo, and Deguest (2014, Example 1, pp 4).

REMARK 1. In the case where the well-diversified portfolio of $\Phi \mathbf{w} \mathbf{R}+\mathbf{y}^{*}$, is obtained by minimization: (i) the inequality $\geq_{2}$ in (5) is replaced by the inequality $\leq$ (Concavity becomes convexity), (ii) the inequality $\geq$ and the function $\mathrm{min}$.(in (6) are replaced by the inequality and $\leq$ the function $\max ($.$) (Quasi-Concavity becomes quasi-convexity), and (iii) the inequality$ $\geq$ in (13) is replaced by the inequality $\leq$.

5The random variables $R_{1}, \ldots, R_{N}$ are said to be exchangeable if and only if their joint distribution $F_{\mathbf{R}} \mathbf{r}$ is symmetric. A well-known example of an exchangeable sequence of random variables is an independent andidentically distributed sequence of random variables. For more details on exchangeable random variables,we refer readers to Aldous (1985). 


\section{Rationalization}

This section studies the rationality of our axioms with respect to rank-dependent expected utility theory (RDEU). The objective is to provide an economic foundation for our axioms.

\section{I Methodology}

Our rationalization strategy does not consist of testing the RDEU function $\left(\mathrm{E}_{u, h}\right.$. against our axioms, because $\mathrm{E} \ell_{\ell, h}$. is not a measure of portfolio diversification. Instead, we examine the compatibility of our axioms with investors' preference for diversification (PFD) in RDEU theory. We proceed in two steps. First, we identify the measure of portfolio diversification at the core of RDEU theory. Our identification procedure is based on the notion of PFD in RDEU theory. There are several notions of PFD in RDEU theory (see De Giorgi and Mahmoud, 2016 a). We consider the notion of PFD introduced by Dekel (1989) and extended later by Chateauneuf and Tallon (2002) and Chateauneuf and Lakhnati (2007) to the space of random variables. Letbe the preference relation overRof a decision maker (i.e., an investor). This notion of PFD is defined as follows.

DEFINITION 1 (PREFERENCE FOR The preference relation $\mathrm{c}$ exhibits DIVERSIFICATION). preference for diversification if for any $R_{i} \in \mathbf{R}$ and $a_{i} \in[0,1], i \in \mathbf{I}_{N}$ such that $\sum^{N} \underline{\underline{N}}_{1} a_{i}=\mathbf{1}$,

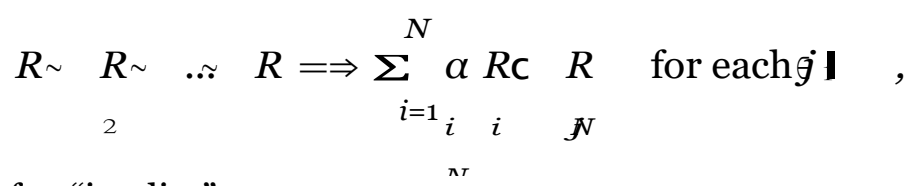

where $=\Rightarrow$ stands for "implies".

Definition 1 states that if assets are equally desirable, then the investor will want to diversify. This notion of PFD is equivalent to the notion of risk aversion in expected utility theory and to the notion of strong risk aversion (i.e. risk aversion in the sense of mean-preserving spread as defined by De Giorgi and Mahmoud (2016 a, Definition 9, pp. 152)) in RDEU theory.

Let $\left.Q_{C_{(u, h)}}(1+R)=C_{\bar{u}, h^{(}} \mathbf{1}\right)(R+$ b a risk measure induced by the certainty equivalent $C$

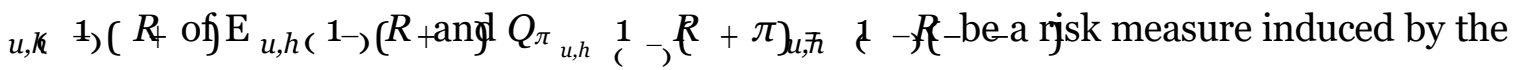
risk premium $\pi_{(u, h)}(1+R) \_$of $\mathrm{E}_{(u, h)}(1+R) . \pi_{(u, h)}(1+R)$ can_be obtained as 
$\pi_{(u, h)}(R)=\mathrm{E}(R)-C_{(u, h)}(R)$. Definition 1 is equivalent to the following.

DEFINITION 2 (PREFERENCE FOR DIVERSIFICATION: EquivalENCE). The preference relation c shows a preference for diversification if for any $R_{i} \in \mathrm{R}$ and $\alpha_{i} \in[0,1], i \in \mathbf{I}_{N}$ such that $\sum_{i=1}^{N} \alpha_{i_{-}}=1$, the following equivalent conditions are satisfied

$$
\begin{aligned}
& \text { (i) } \mathrm{E}_{(u, h)}\left(1+R_{1}\right)=\ldots=\mathrm{E}_{(u, \bar{h})}\left(1+R_{N}\right) \Longrightarrow \mathrm{E}_{(u, \bar{h})}\left(1+\sum_{\tau^{1}}^{N} \alpha_{i} R_{i}\right) \geq \mathrm{E}_{(u, \bar{h}}\left(1+R_{j}\right) \quad \text { for each } j \in \\
& \mathbf{I}_{N} \\
& \text { (ii) } Q_{(u, \bar{h})}\left(1+R_{1}\right)=\ldots=Q_{C} \quad{ }_{(u, \bar{h})}\left(1+R_{N}\right)=\Rightarrow Q_{C}{ }_{(u, \bar{h})}\left(1+\sum_{\tilde{i} 1}^{N} \alpha_{i} R_{i}\right) \leq Q_{C} \quad(u, \bar{h})(1+ \\
& R_{j} \text { ) for each } j \in \mathrm{I}_{N} \\
& \text { (iii) } Q_{\pi_{(u, \hbar)}}\left(1+R_{1}\right)=\ldots=Q_{\pi} \quad{ }_{(u, \hbar)}\left(1+R_{N}\right)=\Rightarrow Q_{\pi} \quad{ }_{(u, \hbar)}\left(1+\sum_{t_{1}}^{N} \alpha_{i} R_{i}\right) \leq Q_{\pi} \quad(u, \hbar)\left(1+R_{j}\right) \quad \text { for each } j \in \\
& \mathbf{I}_{N} \text {. }
\end{aligned}
$$

Because diversification is a risk reduction tool, we focus on parts (ii) and (iii) of Definition 2. Multiplying the inequality in (ii) and (iii) by $\alpha_{j}$ and summing over $j$, we obtain

$$
Q_{l}\left(1+\sum_{i=1}^{N} a_{i} R_{i}\right) \leq \sum_{i=1}^{N} a_{j} Q_{l}\left(1+R_{j}\right) \text { for each } l \in\left\{C_{(u, \bar{h})}, \pi_{(u, \bar{n})}\right\} .
$$

From (24), in line with Embrechts, Furrer, and Kaufmann (2009) (see item (iii) on page 29), the gain of diversification in the RDEU theory can be measured by the difference

$$
Q_{l}(\mathbf{w} \mid \mathbf{R})=\sum_{i=1}^{N} w_{i} Q_{l}\left(\mathbf{1}+R_{i}\right)-Q_{l}\left(\mathbf{1}+\sum_{i=1}^{N} w_{i} R_{i}\right) \text { for each } l \in\left\{C_{(u, h)},-\pi \pi_{(u, h)}\right\} .
$$

Next, we test the identified measure $Q_{l}(\mathbf{w} \mid \mathbf{R})$ against our axioms. We distinguish three cases: (i) $h($.) linear i.e. $h(h)$ ) $p$; (ii) $u$. (linear i.e. $u x$ ( $x$ ) and (iii) $h$. and $(y$ non linear. The case $h$. and $u$.) linear (s) ignored because it is irrelevant for risk aversion. In each case, we assume that $u$. is concave and $h \cdot \mathrm{is}$ (gonvex or $h$. iळ)concave, to be consistent with our considered notion of preference for diversification (see De Giorgi and Mahmoud, 2016 a, Proposition 13). If the identified measure $Q_{l}\left({ }^{\mathbf{w}} l^{\mathbf{R}}\right)$ satisfies our axioms, we consider that our axioms are rationalized by RDEU theory. Our definition of compatibility of our axioms with PFD in RDEU theory is therefore as follows. 
DEFINITION 3 (COMPATIBILITY WITH PFD IN REDU THEORY). Our axioms are compatible with PFD in RDEU theory if and only if they are satisfied by $Q_{C_{u, \hbar)}}(\mathbf{w} \mid \mathbf{R})$ or $Q_{\pi_{(u, \hbar)}}(\mathbf{w} \mid \mathbf{R})$.

\subsection{Results}

\subsection{I $h($.)linear: Expected Utility Theory}

Let us first study the rationality of our axioms with respect to RDEU theory in the case where $h_{(\cdot)}$ is linear. In that case, RDEU theory reduces to expected utility (EU) theory. More formally,

$$
\mathrm{E}_{(u, h)}(1+R)=\mathrm{J} \underset{-\infty}{\infty} u(1+r) d F_{R}(r)
$$

Two cases are considered: small risk in the sense of Pratt (1964) and the location-scale family of distributions.

4.2.I.I Risk is small In this case, we assume that risk is small in the sense of Pratt (1964) i.e. is measured by variance $\left(\sigma_{i}^{2}\right.$, for each $\left.i \in \mathrm{I}_{N}\right)$. It follows that the approximation of the local risk premium of $u($.$) is \mu^{u}(X) \quad{ }^{2}{ }^{1} \operatorname{Var}(X) k(1+\mathrm{E}(X))$, where $k(x)=-{ }^{u}$ "(x) the measure of local risk aversion of $u$ (.)in a small risk scenario. It follows that $Q_{C_{u}}(\mathbf{w} \mid \mathbf{R})=$ $-Q_{\pi_{u}}(\mathbf{w} \mid \mathbf{R})=\underline{\underline{R}}^{2}\left(\sum_{=1}^{\underline{i}^{i}} w_{i} \sigma^{2} k\left(1+\mu_{i}\right)-\sigma^{2}(\mathbf{w}) k(1+\mu(\mathbf{w}))\right)$.

We refer to this compatibility as local compatibility. The following proposition establishes the necessary and sufficient conditions.

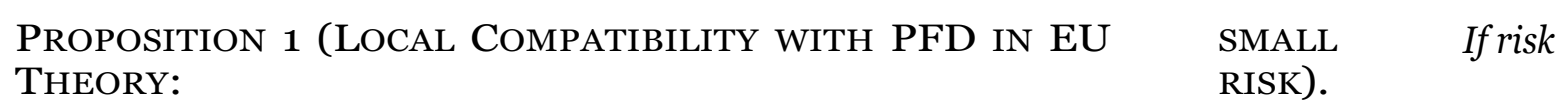
is small, then $Q(\mid \mathbf{w}) \mathbf{R}_{u}$ satisfies our axioms if and only if the absolute risk aversion of $u($.$) is constant; formally, k(x)=-u^{\prime \prime}(x)=c, c \in R$, where $u^{\prime}($.$) and u^{\prime \prime}($.$) are the first and$ second derivatives of $u($.$) .$

From Proposition 1, we therefore have the following result.

COROLLARY 1 (RATIONALIZATION WITH RDEU THEORY:

rationalized by RDEU theory if $\mathrm{h}$. (2)linear, risk is small and the absolute risk aversionof $u($.$) is constant.$
SMALL Ouraxioms are RISK). 
The negative exponential utility function, $y=x-\exp (\lambda x$, )with $\lambda$ o the investor's riskaversion coefficient, is the only known example of a concave utility function that implies constant absolute risk aversion. Thus, if risk is small, our axioms can be rationalized by RDEU theory if and only if $u($.$) is the negative exponential utility function.$

4.2.I.2 Location-Scale Family of Distributions In the second case, we assume that each distribution of asset returns belongs to the location-scale family. This family of distribu- tions includes the normal distribution, the student's t-distribution and all other elliptical distributions. For more details see Meyer (1987). The following proposition establishes the necessary and sufficient conditions.

PROPOSITION 2 (COMPATIBILITY WITH PFD IN EU THEORY: LOCATION-SCALE FAMILY). If each asset return distribution belongs to the location-scale family, then $Q{ }_{C_{u}}(\mathbf{w} \mid \mathbf{R})$ satisfies our axioms if and only if the certainty equivalent has the following additive separable form

$$
C_{u}(R)=\mu-g(\sigma)
$$

for a strictly increasing continuous and homogeneous function $g($.$) on \mathrm{R}_{+}$.

From Proposition 2, we therefore have the following result.

COROLLARY 2 (RATIONALIZATION WITH RDEU THEORY:
LOCATION-SCALE FAMILY).
Our

axioms are rationalized by $R D E U$ theory if each asset return distribution belongs to the location-scale family and the certainty equivalent has the additive separable form in (27).

Below, we present an example of location-scale distribution and utility function for which Proposition 2 is valid.

ExAmple 3 (Normal Distribution and Negative Exponential Utility). Assume that the asset returns are normally distributed and the utility function is negative exponential. One can show that

$$
C_{u}(R(\mathbf{w}))=\mathbf{w}^{\mathrm{T}} \boldsymbol{\mu}-\lambda \sigma^{2}(\mathbf{w}) .
$$

Proposition 2 also implies that our axioms can be rationalized by the additive separable 
mean-variance utility functions (including Markowitz's (1952) mean-variance utility) axiomatized by Nakamura (2015, Theorem 4., pp. 544).

Propositions 1 and 2 jointly represent the necessary and sufficient conditions of our axioms to be compatible with PFD in EU theory. In Propositions 1 and 2, we have compatibility only when risk is measured by variance, so the conditions might be thought to be restrictive, thereby considerably weakening the desirability of our axioms. However, this is not the case, because the majority of our axioms remain compatible with PFD in EU theory when we consider other standard utility functions. For example, exploiting the results in Müller (2007), if we consider the negative exponential utility with a non-Location-Scale family of distributions, one can verify that $Q_{C_{u}}(\mathbf{w} \mid \mathbf{R})$ satisfies all the axioms except Homogeneity. In the case of the power or logarithmic utility function, one can verify that $Q_{C_{u}}(\mathbf{w} \mid \mathbf{R})$ satisfies all the axioms, except Concavity, Quasi-Concavity, Translation Invariance and Homogeneity. Moreover, as we show in the next two subsections, our axioms are also relevant when risk is not completely captured by the variance.

\subsection{2 u(.)linear:Yaari's (1987) Dual Utility Theory}

Now, assume that $(1)$ is linear i.e. $\{x \neq x$. In that case RDEU theory reduces to Yaari's (1987) dual utility (DU) theory. More formally

$$
\mathrm{E}_{(u, h)}(1+R)=\mathrm{J} \underset{-\infty}{\infty}(1+r) d h\left(F_{R}(r)\right) .
$$

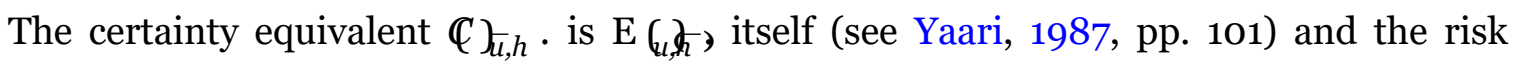
premium is $\pi_{(u, h)}(1+R)=\mathrm{E}(1+R)-\mathrm{E}_{(u, h)}(1+R)$ (see Denuit, Dhaene, and Van Wouwe, 1999).

Proposition 3 examines the compatibility of our axioms with PFD in DU theory.

PRoposition 3 (COMPATIBILITy WITH PFD IN DU THEORY). Our axioms are satisfied by $Q_{C_{(u, h)}}(\mathbf{w} \mid \mathbf{R})$ and $Q_{\pi_{(u, h)}}-(\mathbf{w} \mid \mathbf{R})$ if and only if $h(\overline{.})$ is convex or $h($.$) is concave.$

From Proposition 3, we therefore have the following result. 
COROLLARY 3 (RATIONALIZATION WITH RDEU THEORY). Ouraxioms are rationalized by $R D E U$ theory if the utility function $u$. Es linear and the distortion function $h$. is $\bar{c}$ brtvexor equivalently the conjugate distortion function $h($.$) is concave.$

\subsection{3 u. . and $h$. ( nonlinear}

Finally, assume that the utility function $u$. ) and the distortion function $\bar{h}$ ( or the conjugate distortion function $h($.$) are nonlinear. In that case, the certainty equivalent is$

$$
C_{(u, \bar{h}}(1+R)=u^{-1}\left(J_{-\infty}^{\infty} u(1+r) d h\left(F_{R}(r)\right)\right) .
$$

The risk premium based on $C_{(u, \hbar)}(1+R)$ is $\pi=E(1+R)-C_{(u, h)}(1+R)$.

Assume further that $u_{(\cdot)}$ and $h_{(}$are continuously differentiable. Then, $\mathrm{E}_{u, \hbar} \ell+R_{\mathfrak{\gamma}}$ can be interpreted as an expected ytility

$$
\mathrm{E}_{(u, h)}(1+R)=\mathbf{J}{ }_{-\infty}^{\infty} u(1+r) h^{\prime}\left(F_{R}(r)\right) d\left(F_{R}(r)\right)=\mathrm{E}(v(1+R)),
$$

with $v(x)=u(x) z^{\prime} F_{x} x($. The results of Subsubsection 4.2.1 (Propositions 1 and 2) can therefore be applied to $\mathrm{E}_{(u, h)}(1+R)$ with $u($.$) and h($.$) or h($.$) nonlinear.$

Applying Proposition 1 to $\mathrm{E}_{(u, h)}(1+R)=\mathrm{E}(v(1+R))$, we obtain Proposition 4 .

PROPOSITION 4 (LOCAL COMPATIBILITY WITH PFD IN RDEU THEORY: SMALL RISK). If risk is small, then $Q \quad(\mathbf{w} \mid \mathbf{R})$ satisfies our axioms if and only if the absolute risk aver$C_{u, h}$

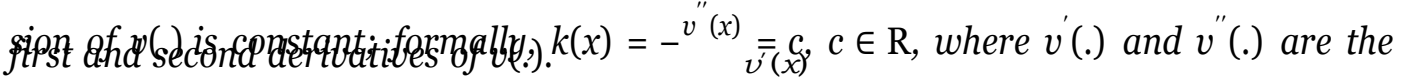

From Proposition 4, we therefore have the following result.

$\begin{array}{ll}\text { COROLLARY } 4 \text { (RATIONALIZATION WITH RDEU } & \text { SMALL } \\ \text { THEORY: } & \text { RISK). }\end{array}$ rationalized by RDEU theory if $\mathrm{u}$. (a)d $h$. afr nonlinear, risk is small and the absoluterisk aversion of $v($.$) is constant.$

Below we present an example of $v($.) with constant absolute risk aversion. 
EXAMPlE $4(v($.$) WITH CONSTANT ABSOLUTE RISK AVERSION). Assume that$

(i) $u($.) is the negative exponential utility function: $u(x)=-\exp (-\lambda x)$ with $\lambda>0$;

(ii) $h\left(\right.$.) is: $h(x)=\frac{\delta^{x} \exp \left(-\beta F_{\bar{R}}^{1}(t)\right) d t}{\int 0^{1} \exp \left(-\beta F R^{1}(t)\right) d t}$ with $\beta>0$ a parameter capturing the degree of concavity of $h(x)$;

One can verify that $h(x)$ is a strictly increasing concave function, and

$$
v(1+r)=u(1+r) h^{\prime}\left(F_{R}(r)\right)=-\exp (-\lambda(1+r)) \exp (-\beta r)=-\exp (-\lambda) \exp (-(\lambda+\beta) r) \text { (32)From }
$$

(32), it is clear that the absolute risk aversion of $v($.$) is constant.$

Applying Proposition 2 to $\mathrm{E}_{(u, h)}(1+R)=\mathrm{E}(v(1+R))$, we obtain Proposition 5 .

PROPOSITION 5 (COMPATIBILITY WITH PFD IN RDEU THEORY: LOCATION-SCALE FAMILY).

If each asset return distribution belongs to the location-scale family, then $Q C_{(u, \hbar)}(\mathbf{w} \mid \mathbf{R})$ satisfies our axioms if and only if the certainty equivalent has the additive separable form in Equation (27).

From Proposition 5, we therefore have the following result.

COROLLARY 5 (RATIONALIZATION WITH RDEU THEORY:

axioms are rationalized by RDEU theory with $u$. (a)d $h$. being nonlinear if each assetreturn distribution belongs to the location-scale family and the certainty equivalent has the additive separable form in (27).

Below, we present an example of location-scale distribution, utility function $(\ell)$ and conjugate distortion function $h($.$) for which Proposition 5$ is valid.

EXAMPle 5 (NORMAL Distribution, $u(x)=-\exp (-\lambda x)$ AND $\left.h(x)=\int^{e} \exp \left(-\beta F^{R_{1}}(t)\right) d t\right)$. Assume that
LOCATION-SCALE Our FAMILY). 
(iii) $R(\mathbf{w})$ is a continuous normal random variable.

From Example 4,

$$
v(1+r)=u(1+r) h^{\prime}\left(F_{R}(r)\right)=-\exp (-\lambda(1+r)) \exp (-\beta r)=-\exp (-\lambda) \exp (-(\lambda+\beta) r)(33) \operatorname{In}
$$

that case, one can show that

$$
C_{(u, \bar{h}}(R(\mathbf{w}))=\mathbf{w}^{\mathrm{T}} \boldsymbol{\mu}-(\lambda+\beta) \sigma^{2}(\mathbf{w}),
$$

where $\lambda+\beta$ is the coefficient of risk aversion.

\section{Existing Diversification Measures}

In this section, we explore whether some useful methods of measuring correlation diversification satisfy our axioms. We consider the four correlation diversification measures used most frequently on the marketplace and by academic researchers in both finance and insurance:

(i) Portfolio variance

$$
\sigma^{2}(\mathbf{w} \mid \mathbf{R})=\mathbf{w}^{\mathrm{T}} \mathbf{\Sigma} \mathbf{w}
$$

(ii) Diversification ratio (DR)

$$
\operatorname{DR}(\mathbf{w} \mid \mathbf{R})=\frac{\sqrt{\mathbf{w}^{\mathrm{T}} \boldsymbol{\sigma}}}{\overline{\mathbf{W} \mathbf{\Sigma} \mathbf{w}}}
$$

(iii) Embrechts, Furrer, and Kaufmann's (2009) class of measures

$$
\mathrm{D}\left(\mathbf{w} \mathbf{R}=\sum_{i=1}^{N} Q w{ }_{i}{ }_{i}-Q \mathbf{w} .\right.
$$

(iv) Tasche's (2006) class of measures

$$
\begin{aligned}
& \operatorname{DR}(\mathbf{w} \mid \mathbf{R})=\frac{Q\left(\mathbf{w}^{\mathrm{T}} \mathbf{R}\right)}{\operatorname{Qi1}_{i{ }_{i}}^{Q(w R)}} . \\
& \text { e } \quad \sum^{N}=
\end{aligned}
$$

Portfolio variance is the risk measure in the mean-variance model. It is usually used to 
quantify the benefit of diversification (Markowitz, 1952, 1959; Sharpe, 1964), and is formally analyzed as a portfolio diversification measure by Frahm and Wiechers (2013).

The diversification ratio (DR) is a diversification measure introduced by Choueifaty and Coignard (2008); see also Choueifaty, Froidure and Reynier (2013). An intuitive interpretation of the DR is the Sharpe ratio when each asset's volatility is proportional to its expected premium, i.e. $\mathrm{E}\left(\boldsymbol{R}_{i}\right) \boldsymbol{R}_{N} \delta \boldsymbol{\theta}_{i}$, for each $\boldsymbol{i}_{N \in \mathrm{Where}} \delta \mathrm{o}$ and $>\boldsymbol{R}_{N}$ is the return of the risk-free asset.

Embrechts, Furrer, and Kaufmann's (2009) class $\left(\mathrm{D}_{e}\right)$ is the class of diversification measures induced by a risk measure $Q$ \}. An intuitive interpretation can be provided for $D_{e}$ when $Q($.$) is homogeneous of degree one. In that case,$

$$
\mathrm{D}_{e}(\mathbf{w} \mid \mathbf{R})=\sum_{i=1}^{N} w_{i}\left(Q\left(R_{i}\right)-Q\left(\mathbf{w}^{\mathrm{T}} \mathbf{R}\right)\right)
$$

The term in parentheses, $Q R\}) Q \mathbf{v}^{\mathrm{T}} \mathbf{R}$, measures the benefit of diversification, in terms of risk reduction, of holding portfolio $\mathbf{w}$ instead of concentrating on single-asset $i$. It follows that $\mathrm{D}_{e}$ quantifies the average benefit of diversification. Tasche's (2006) class of diversification measures $\left(\mathrm{DR}_{\ell}\right)$ is a normalized version of $\mathrm{D}_{\ell}$. Some authors (see Degen, Lambrigger, and Segers, 2010; Mao, Lv, and $\mathrm{Hu}, 2012)$ refer to $\mathrm{DR}_{\ell}$ as a measure of concentration risk and $1-\mathrm{DR}_{e}$ as a measure of diversification benefit.

The following proposition analyzes these four measures in light of our axioms.

Proposition 6 (COMPATIBILITY WITH EXISTING DIVERSIFICATION MEASURES). The fol-lowing statements hold.

(i) Portfolio variance satisfies our axioms if and only if assets have the same standard deviation i.e $\sigma_{i} \bigoplus_{j}, i, j 1, \ldots, N$.

(ii) The diversification ratio satisfies our axioms.

(iii) Embrechts, Furrer, and Kaufmann's (2009) class of measures satisfies our axioms if and only if $Q$. is cor(u)ex (or quasi-convex), positively homogeneous, translation invariant and reverse lower comonotonic additive.

(iv) Tasche's (2006) class of measures satisfies our axioms if and only if $Q($.$) is convex$ 
(or quasi-convex), positively homogeneous, translation invariant and reverse lower comonotonic additive.

Part (i) of Proposition 6 shows that the portfolio variance satisfies our axioms, but under very restrictive (if not impossible) conditions that all assets have identical variances. More specifically, portfolio variance satisfies our axioms, except Size Degeneracy, Risk Degeneracy and Reverse Risk Degeneracy. This result, rather than weakening our axioms, reveals the limits of portfolio variance as an adequate measure of diversification in the mean-variance model.

Part (ii) of Propositions 6 shows that DR is a coherent correlation diversification measure. Since the DR coincides with the Sharpe ratio when each asset's volatility is proportional to its expected premium, this result implies that the Sharpe ratio is a coherent correlation diversification measure under the above proportionality condition. It is also straightforward to verify that if the Sharpe ratio is a coherent correlation diversification measure, then each asset's volatility is proportional to its expected premium. As a result, the Sharpe ratio is a coherent correlation diversification measure if and only if each asset's volatility is proportional to its expected premium.

Parts (iii) and (iv) show that Embrechts, Furrer, and Kaufmann's (2009) and Tasche's (2006) classes of measures are also coherent, under the conditions that the risk measure $Q$ (.) is convex (or quasi-convex), homogeneous, translation invariant and reverse lower comonotonic additive. ${ }^{6}$

Because Embrechts, Furrer, and Kaufmann's (2009) and Tasche's (2006) classes of measures are diversification measures induced by a risk measure, parts (iii) and (iv) also establish the conditions under which a coherent risk measure induces a coherent diversification measure.

COROLLARY 6 (COHERENT RISK MEASURE (ARTZNER ET AL, 1999)). A coherent risk measure induces a coherent diversification measure if and only if the coherent risk measure is reverse lower comonotonic additive.

6Embrechts, Furrer, and Kaufmann's (2009) and Tasche's (2006) classes of measures satisfy our axioms under the same conditions because the Tasche's (2006) class of measures is a normalized version of the Embrechts, Furrer, and Kaufmann's (2009) class of measures. 
Corollary 6 follows from the fact that any coherent risk measure is convex, positively homogeneous (with $\kappa$ 1), translation invariant and all coherent risk measures are not reverse lower comonotonic additive. An example of a coherent risk measure that is not reverse lower comonotonic additive is the expectation risk measure, i.e. $(Q) X=E X$. An example of a coherent risk measure that is reverse lower comonotonic additive is any concave distortion risk measure, as implied by Proposition 3. It follows that the Expected Shortfall, which is chosen over Value-at-Risk in Basel III (see BCBS, 2013), induces a coherent diversification measure in the case of continuous distributions. However, the Conditional Value-at-Risk (see Rockafellar and Uryasev, 2000, 2002) induces a coherent diversification measure without restrictions.

Parts (iii) and (iv) of Propositions 6 also imply that the family of deviation risk measures of Rockafellar, Uryasev, and Zabarankin (2006) induces a family of coherent diversification measures, but not the family of convex risk measures (see Föllmer and Schied, 2002; Frittelli and Gianin, 2002, 2005) and Aumann and Serrano's (2008) risk measure. Part (iv) of Propositions 6 also supports the findings of Salazar Flores et al (2017) that the diversification delta of Vermorken, Medda, and Schroder (2012) is an inadequate measure of portfolio diversification.

The fulfillment of our axioms by the diversification ratio, Embrechts, Furrer, and Kaufmann's (2009) and Tasche's (2006) classes of measures, strengthens our axioms. They are relevant to a number of measures with which we have considerable experience. In turn, several desirability properties of these measures can be rationalized by rank-dependent expected utility theory, and their popular use in empirical works and on the marketplace can be defended by our axioms.

\section{Representation}

To close this paper, we examine whether or not our axioms imply a family of representations. As mentioned in Section 3, from Marshall, Olkin, and Arnold (2011, C.2. and C.3. Propositions, pp 97-98), Symmetry and Concavity or Quasi-Concavity taken together imply that $\Phi(\mathbf{w} \mid \mathbf{R})$ is Schur-concave in $\mathbf{w}$ when the sequence $R_{1}, \ldots, R_{N}$ is exchangeable. There- 
fore, Marshall, Olkin, and Arnold (2011, B.1. Proposition, pp 393) provides the following representation form of $\Phi(\mathbf{w} \mid \mathbf{R})$

$$
\Phi(\mathbf{w} \mid \mathbf{R})=\mathrm{E}(\varphi(\mathbf{w}, \mathbf{R}))
$$

where $\varphi(\mathbf{w}, \mathbf{R})$ satisfies Size Degeneracy, Risk Degeneracy, Reverse Risk Degeneracy, Duplication Invariance, Size Monotonicity, Translation Invariance, Homogeneity, and the following additional properties

(1) $\varphi(\mathbf{w}, \mathbf{R})$ is concave in $\mathbf{w}$ for each fixed $\mathbf{R} \in \mathbf{R}^{N}$.

(2) $\varphi(\boldsymbol{\Pi} \mathbf{w}, \boldsymbol{\Pi} \mathbf{R})=\varphi(\mathbf{w}, \mathbf{R})$ for all permutation matrices $\boldsymbol{\Pi}$.

(3) $\varphi(\mathbf{w}, \mathbf{R})$ is Borel-measurable in $\mathbf{R}$ for each fixed $\mathbf{w}$.

Properties (1) and (2) ensure that $\varphi(\mathbf{w}, \mathbf{R})$ is concave in $\mathbf{w}$ and symmetric in $\mathbf{w}$ when $\mathbf{R}$ is exchangeable, therefore satisfying Concavity and Symmetry. Below, we present two examples of $\varphi(\mathbf{w}, \mathbf{R})$.

EXAMPLE 6 (RAO'S QUADRATIC Consider $\varphi(\mathbf{w}, \mathbf{R})$ such that ENTROPY).

$$
\varphi(\mathbf{w}, \mathbf{R})=\sum_{i, j=1}^{N}\left|\left(R_{i}-\mu_{i}\right)-\left(R_{j}-\mu_{j}\right)\right|^{q} w_{i} w_{j}, 0<q \leq 2
$$

Obviously, $\varphi(\mathbf{w}, \mathbf{R})$ satisfies properties (1) to (3), Size Degeneracy, Risk Degeneracy, Reverse Risk Degeneracy, Duplication Invariance, Size Monotonicity, Translation Invariance, and Homogeneity. As a consequence $\Phi \mathbf{w} \mathbf{R}=\mathrm{E} \varphi(\mathbf{w}, \mathbf{R}))$ is a coherent class of portfolio diversification measures analyzed by Carmichael, Koumou, and Moran (2015) and Carmichael, Koumou, and Moran (2018) under the name Rao's Quadratic Entropy. In the case where $q \bumpeq, \Phi, \boldsymbol{w} \mid \mathbf{R}$ ) coincides with the diversification returns, a popular correlation diversification measure analyzed by Willenbrock (2011), Chambers and Zdanowicz (2014), Bouchey et al (2012), Qian (2012) and Fernholz (2010) under the name excess growth rate.

EXAmple 7 (Diversification MEASure IndUCEd by Expected ShortFall). 
Consider $\varphi(\mathbf{w}, \mathbf{R})$ such that

$$
\varphi(\mathbf{w}, \mathbf{R})=\sum_{i, j=1}^{N} w \operatorname{ESF}(R)-\operatorname{ESF}\left(\mathbf{w}^{\top} \mathbf{R}\right), p \in(0,1),
$$

where $\mathrm{ESF}_{p} X$ max $R_{i} \quad \operatorname{VaR}_{p} \quad R_{i}$, o is the Expected Shortfall of $X$ at level $p$ with $\operatorname{VaR}_{p} X$ the $($ galue-at-risk of $X$ at level $p$. We can verify that $\varphi \mathbf{w}, \mathbf{R}$ safisfies)prop-erties (1) to (3), Size Degeneracy, Risk Degeneracy, Reverse Risk Degeneracy, Duplication Invariance, Size Monotonicity, Translation Invariance, and Homogeneity. As a consequence $\Phi(\mathbf{w} \mid \mathbf{R})$ E $\varphi(\mathbf{w}, \mathbf{R}$ wjith $\varphi \mathbf{w},(\mathbf{R}$ as) defined in (37) is a coherent class of portfolio diversification measures, a member of the family of Embrechts, Furrer, and Kaufmann's (2009) class of measures.

Is the representation form in (35) unique? The following example provides evidence that it is not. It presents a diversification measure that satisfies our axioms, but does not have the representation form (35).

EXAMPlE 8 (DIVERSIFICATION MEASURE INDUCED By STANDARD VOLATILITY). Consider $\Phi(\mathbf{w} \mid \mathbf{R})$ such that

$$
\Phi(\mathbf{w} \mid \mathbf{R})=\mathbf{w}^{\mathrm{T}} \boldsymbol{\sigma}-\sigma(\mathbf{w})
$$

It is straightforward to verify that $\Phi(\mathbf{w} \mathbb{R})$ in (38) satisfies our axioms, but does not have the representation form (35).

In sum, we have the following representation theorem.

PROPOSITION 7 (REPRESENTATION If $\Phi(\mathbf{w} \mid \mathbf{R})$ satisfies our axioms, then $\Phi(\mathbf{w} \mid \mathbf{R})$ THEOREM).

can take the following representation form

$$
\Phi(\mathbf{w} \mid \mathbf{R})=\mathrm{E}(\varphi(\mathbf{w}, \mathbf{R}))
$$

where $\varphi(\mathbf{w}, \mathbf{R}$ fatisfies Size Degeneracy, Risk Degeneracy, Reverse Risk Degeneracy, Dupli- cation Invariance, Size Monotonicity, Translation Invariance, Homogeneity and properties (1) to (3). 


\section{Concluding Remarks and Future Research}

This paper provides an axiomatic foundation for the measurement of correlation diversification in a one-period portfolio theory under the assumption that the investor has complete information about the joint distribution of asset returns. We have specified a set of minimum desirable axioms for measures of correlation diversification, and named the measures satisfying these axioms coherent diversification measures.

We have provided an economic justification of our axioms by analyzing their rationality with respect to rank-dependent expected utility theory. We have distinguished three cases, and we have shown that

(i) if the utility function $u$. Js nonlinear and concave and the distortion function $\bar{h}$ (i\$ linear (in which case rank-dependent expected utility theory reduces to expected utility theory), then our axioms are rationalized by rank-dependent expected utility theory if and only if one of the following conditions is satisfied:

(a) risk is small in the sense of Pratt (1964) and absolute risk aversion is constant (see Proposition 1);

(b) each distribution of asset returns belongs to the location-scale family (including the elliptical distributions) and the certainty equivalent has a particular additive-separable form (see Proposition 2);

(ii) if the utility function is linear and the distortion function is nonlinear (in which case rank-dependent expected utility theory reduces to Yaari's (1987) dual theory) our axioms are rationalized by rank-dependent expected utility theory if and only if the distortion function is convex (see Proposition 3); and

(iii) if the utility and the distortion functions are nonlinear and are continuously differentiable, then our axioms are rationalized by rank-dependent expected utility theory if and only if one of the following conditions is satisfied:

(a) risk is small in the sense of Pratt (1964) and absolute risk aversion of the utility

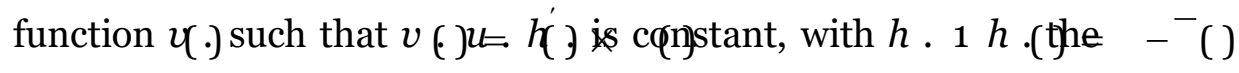
conjugate distortion function (see Proposition 4);

(b) each distribution of asset returns belongs to the location-scale family (includ- 
ing the elliptical distribution), and the certainty equivalent of $($ ) has a particular additive-separable form (see Proposition 5);

These results provide the decision-theoretic foundations of our axiomatic system, and consequently strengthen their economic desirability, reasonableness and relevance.

We have explored whether portfolio diversification measures such as portfolio variance, diversification ratio, Embrechts, Furrer, and Kaufmann's (2009) class of diversification measures and Tasche's (2006) class of diversification measures, which are used in the academic literature and on the marketplace to manage millions of US dollars, satisfy those axioms. We have shown that

(i) portfolio variance satisfies our axioms, under the very restrictive (if not impossible) condition that the assets have identical variance;

(ii) the diversification ratio satisfies our axioms;

(iii) Embrechts, Furrer, and Kaufmann's (2009) and Tasche's (2006) classes of diversification measures satisfy our axioms, under the conditions that the underlying risk measure is convex (or quasi-convex), homogeneous, translation invariant and reverse lower comonotonic additive.

These results strengthen both the axioms and such measures as the diversification ratio and Embrechts, Furrer, and Kaufmann's (2009) and Tasche's (2006) classes of diversification measures. However, they reveal the limits of portfolio variance as an adequate measure of diversification in the mean-variance model.

Finally, we have investigated whether our axioms have functional representations. We have shown that our axioms imply a non-unique family of representations.

Our objective is to offer the first step toward a rigorous theory of correlation diversification measures. We believe that with our axiomatic system this goes in the good direction. Feasible and desirable extensions for future research are

(i) to re-examine the rationality of our axioms considering other risk measures in rankdependent expected utility theory such as the optimal expected utility risk measures 
as in the study by Geissel, Sass, and Seifried (2018) and the extreme risk aggregation approach as in the study by Chen and Hu (2019);

(ii) to extend the rationality of our axioms to cumulative prospect theory;

(iii) to investigate what axioms could be added or relaxed in order to provide a unique family of representations because our axiomatic system does not.

ACKNOWLEDGEMENTS. We thank our discussants, Tan Wang at the 31st Annual Conference of the Northern Finance Association (NFA), Dominic Cervicek at the 7 th Paris Financial Management Conference, and Sebastian Schluetter at the 46th Seminar of the European Group of Risk and Insurance Economists (EGRIE), as well as participants at the 59e congrès annuel de la Société Canadienne de Sciences Économiques and the 53rd Annual Conference of the Canadian Economics Association. This paper is based on material from the first author's dissertation in the Department of Economics at Laval University. We gratefully acknowledges the financial support of "Fonds de Recherche du Québec-Société et Culture" and the Canada Research Chair in Risk Management. 


\section{A Appendix: Proofs}

\section{A.I Proposition 1}

Assume that risk is small. According to Pratt (1964), the approximation of the local risk premium of $u($.$) is \pi_{u}(X) \quad \frac{1}{2} \operatorname{Var}(X) k(1+\mathrm{E}(X))$, where $k(x)=-{ }^{u} \frac{(x)}{u^{\prime}(x)}$ is the _measure of local risk aversion of $u($.$) in a small risk scenario. It follows that Q_{\mathbb{\Theta}}(\mathbf{w} \mid \mathbf{R})=-Q_{\pi_{u}}(\mathbf{w} \mid \mathbf{R})=$

$\underline{\underline{e}}\left(\sum_{=1}^{\dot{N}} w_{i} \sigma^{i} k\left(1+\mu_{i}\right)-\sigma^{2}(\mathbf{w}) k(1+\mu(\mathbf{w}))\right)$. Now let us show that $Q_{C_{u}}(\mathbf{w} \mid \mathbf{R})$ satisfies our axioms if and only if $k x$ is a constant function.

\section{A.I.I Sufficiency}

Suppose that $k(x)=c, c>0$. Then $Q_{C_{u}}(\mathbf{w} \mid \mathbf{R})=c\left(\mathbf{w}^{\mathrm{T}} \boldsymbol{\sigma}^{2}-\sigma^{2}(\mathbf{w})\right)$. Therefore, we consider $\underline{Q}_{C_{u}}(\mathbf{w} \mid \mathbf{R})=\mathbf{W}^{\mathrm{T}} \boldsymbol{\sigma}^{2}-\sigma^{2}(\mathbf{w})$ for the proof.

(C)- Since $\sigma^{2}(\mathbf{w})$ is convex on $\mathrm{W}, \underline{Q}_{C_{u}}(\mathbf{w} \mid \mathbf{R})$ is concave on $\mathrm{W}$.

(SD)- It is straightforward to verify that $\underline{Q}_{C_{u}}\left(\boldsymbol{\delta}_{i} \mid \mathbf{R}\right)=\sigma_{i}^{2}-\sigma_{i}^{2}=0=\Phi$, for each $i \in \mathbf{I}_{N}$.

(RD)- Since $A_{i}=A, R_{i}=R$ for each $i \in \mathrm{I}_{N}$. Then, $\sigma_{i}=\sigma_{j}=\underline{\sigma}$ and $\rho_{i j}=1$ for each $i, j \in \mathrm{I}_{N}$ with $\underline{\sigma}>$ o. It follows that $\underline{Q}_{C_{u}}(\mathbf{w} \mid \mathbf{R})=\underline{\sigma}^{2}-\underline{\sigma}^{2}\left(\sum_{i=1}^{N} w_{i}\right)^{2}=0=\underline{\Phi}$.

(RRD)- Since $w_{i} \geq 0$, for each $i \in \mathbf{I}_{N}$ and $\underline{Q} \quad(\mathbf{w} \mid \mathbf{R})=\sum_{=1}^{N} w_{i}\left\|R_{i}-R(\mathbf{w})\right\|^{2}, \underline{Q} \quad(\mathbf{w} \mid \mathbf{R})=$ $0 \Leftrightarrow R_{i}=R(\mathbf{w})$, for each $i \in \mathrm{I}_{N}$. The result follows.

(DI)- Since $A_{N+1}^{\dagger}=A_{k}, A_{i}^{+}=A_{i}$ for each $i \in \mathbf{I}_{N}$,

$$
\begin{aligned}
& \left.\underline{Q} \quad \mathbf{w}_{\mathrm{A}} \mid \mathbf{R}_{\mathrm{A}}\right)=\Sigma w \quad \sigma_{+}-\Sigma w+{ }_{A_{i}}+\sigma+\sigma+ \\
& C_{u}\left(+\quad+\quad{ }_{i=1}^{H \neq 1} \quad A_{i} A_{i} \quad N_{i j=1}^{+1} A_{i} \quad A_{j} A_{i} A_{j}\right. \\
& =\sum_{i \neq k=1}^{N-1} w_{A_{i}}^{+A_{i}} A_{A_{N+1}}+\left(\begin{array}{llll}
w_{A_{k}} & + & A_{k}
\end{array}\right. \\
& \mathrm{N}-2 \\
& -\underset{i, j \neq k=1}{\grave{j}} w_{A_{i}^{+}} w_{A_{j}^{+}} \sigma_{A_{i}^{+}} \sigma \sum w_{j_{i=1}} A_{i}^{+}\left(w A_{k}^{+}+w_{A_{N+1}^{+}}\right) \sigma_{A_{i}^{+}} \sigma_{A_{k}^{+}} .
\end{aligned}
$$

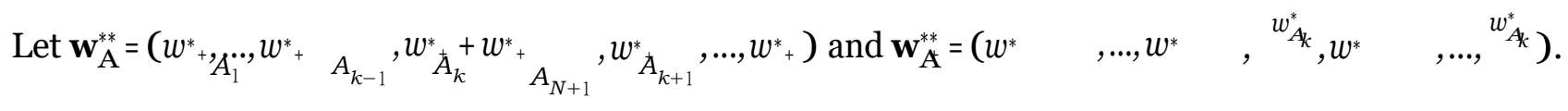

$$
A_{N} \quad A_{1} \quad A_{k-1} \quad \overline{2} \quad A_{k+1} \quad \overline{2}
$$


It follows that

$$
\begin{gathered}
\underline{Q}_{C}\left(\mathbf{w}_{\mathrm{A}^{+}}^{*} \mid \mathbf{R}_{\mathrm{A}^{+}}\right)=Q_{-C_{u}}^{Q}\left(\mathbf{w}_{\mathrm{A}}^{* *} \mid \mathbf{R}_{\mathrm{A}}\right) \leq \underline{-C}_{C_{u}}\left(\mathbf{w}_{\mathrm{A}}^{*} \mid \mathbf{R}_{\mathrm{A}}\right), \\
\underline{Q}_{C_{u}}\left(\mathbf{w}_{\mathrm{A}}^{*} \mid \mathbf{R}_{\mathrm{A}}\right)=_{-C}\left(\mathbf{w}_{\mathrm{A}}^{* *} \mid \mathbf{R}_{\mathrm{A}^{+}}\right) \leq \underline{Q}_{-C}\left(\mathbf{w}_{\mathrm{A}^{+}}^{*} \mid \mathbf{R}_{\mathrm{A}^{+}}\right) .
\end{gathered}
$$

Then

$$
\begin{aligned}
\underline{Q}_{C_{u}}\left(\mathbf{w}_{\mathrm{A}^{+}}^{*} \mid \mathbf{R}_{\mathrm{A}^{+}}\right) & =\underline{Q}_{-C_{u}}\left(\mathbf{w}_{\mathrm{A}}^{*} \mid \mathbf{R}_{\mathrm{A}}\right) \\
w_{A_{i}}^{*} & =w_{A_{i}^{*}}^{*}, \text { for each } i \neq k, i \in \mathbf{I}_{N} \\
w_{A_{k}}^{*} & =w_{A_{k}^{+}}^{*}+w_{A_{N^{+}+1}^{*}}^{*} .
\end{aligned}
$$

(M)- Consider a portfolio $\mathbf{w}_{\mathbf{A}^{++}}=\left(\mathbf{w}_{\mathbf{A}}^{*}, 0\right)$. Portfolio $\mathbf{w}_{\mathbf{A}^{++}}$is an element of $\mathbf{W}_{\mathbf{A}^{++}}^{N+1}$, so $\underline{Q}_{C_{u}}\left(\mathbf{w}_{\mathrm{A}^{+}}^{*} \mid \mathbf{R}_{\mathrm{A}^{++}}\right) \geq Q_{-C_{u}}\left(\mathbf{w}_{\mathrm{A}^{++}} \mid \mathbf{R}_{\mathrm{A}^{++}}\right)$. Since $\underline{Q}_{-C_{u}}\left(\mathbf{w}_{\mathrm{A}^{++}} \mid \mathbf{R}_{\mathrm{A}^{++}}\right)=\underline{Q}_{-C_{u}}\left(\mathbf{w}_{\mathrm{A}}^{*} \mid \mathbf{R}_{\mathrm{A}}\right), \underline{Q}_{C_{u}}\left(\mathbf{w}_{\mathrm{A}^{+}}^{*} \mid\right.$ $\left.\mathbf{R}_{\mathrm{A}^{++}}\right) \geq \underline{C}_{C_{u}}\left(\mathbf{w}_{\mathrm{A}}^{*} \mid \mathbf{R}_{\mathrm{A}}\right)$.

(TI,H)- Because covariance is translation invariant and homogeneous of degree two.

(S)- Since $\sigma_{i}=\sigma_{j}=\underline{\sigma}$ and $\rho_{i j}=\varrho$ for each $i, j \in \mathrm{I}_{N}$ when $R_{1}, \ldots, R_{N}$ is exchangeable,

$\underline{Q}_{C_{u}}(\mathbf{w} \mid \mathbf{R})=\underline{\sigma}^{2}-\underline{\sigma}^{2}\left(\sum_{i=1}^{N} w_{i}^{2}+\underline{\rho} \sum_{i, j=1}^{N} w_{i} w_{j}\right)$. It is straightforward to verify that $\underline{Q_{C_{u}}}(\mathbf{w} \mid \mathbf{R})$ is symmetric.

\section{A.I.2 Necessity}

For the converse, suppose that $Q_{C_{u}}(\mathbf{w} \mid \mathbf{R})$ satisfies our axioms and let us show that $k(x)$ is a constant function. To do so, we proceed by contradiction. Suppose that $k(x)$ is not a constant function. It is straightforward to verify that $Q_{C_{u}}(\mathbf{w} \mid \mathbf{R})$ satisfies translation invariance and homogeneity if and only if $k(1+x)$ is translation invariant and homogeneous, which is the case only if $k(x)$ is a constant function. This contradicts our hypothesis that $k(x)$ is constant. As a consequence, $Q_{C_{u}}(\mathbf{w} \mid \mathbf{R})$ satisfies our axioms, which implies that $k(x)=c, c>0$. 


\section{A.2 Proposition 2}

\section{A.2.I Sufficiency}

Follows from the proof of the sufficiency part of Proposition 1.

\section{A.2.2 Necessity}

Since asset $i$ return distributions belong to the location-scale family, from Meyer (1987), $C_{u}(R)=u^{-1}(U(\mu, \sigma))$, where $\mathrm{E}_{u}(R)=U(\mu, \sigma)=\int u(\mu+\sigma x) d x$ and $u^{-1}($.$) is the inverse$ of $u(\cdot)$. It is obvious that if $Q_{C_{u}}(\mathbf{w} \mid \mathbf{R})$ satisfies our axioms, then $C_{u}(R)=u^{-1}(U(\mu, \sigma))=$ $\mu-g(\sigma)$ with $g$ ( js a strictly increasing, continuous and homogeneous function on $\mathrm{R}_{+}$.

\section{A.3 Proposition 3}

We focus only on $Q_{C_{\hbar}}(\mathbf{w} \mid \mathbf{R})$.

\section{A.3.I Sufficiency}

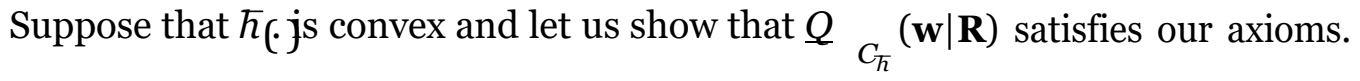

(C)- Since $\bar{h}\left(\right.$.) is convex, $C_{\hat{h}}^{-(.)}$is convex on R (Tsanakas and Desli, 2003). It follows that $C_{\bar{h}}(\mathbf{w} \mid \mathbf{R})$ is convex on $\mathrm{W}$ and consequently, $Q_{C_{\bar{h}}}(\mathbf{w} \mid \mathbf{R})$ is concave.

(SD)- Let $\boldsymbol{\delta}_{i} \in \mathrm{W}$ be a single-asset $i$ portfolio. It is straightforward to show that $Q_{C_{\bar{h}}}\left(\boldsymbol{\delta}_{\boldsymbol{i}} \mid \mathbf{R}\right)=$ $C_{h}\left(R_{i}\right)-C_{h}\left(R_{i}\right)=\mathrm{O}=\underline{\Phi}$.

(RD)- Since $A_{i}=A, R_{i}=R$ for each $i \in \mathrm{I}_{N}$. Then, $Q_{C_{\bar{h}}}\left(R_{i}\right)=Q_{C_{\bar{h}}}\left(R_{j}\right)$ for each $i, j \in \mathrm{I}_{N}$. It follows that $\underline{Q}_{C_{h}}(\mathbf{w} \mid \mathbf{R})=C_{h}(R)-C_{h}(R)=0=\underline{\Phi}$.

(RRD)- Since $C_{h}(R)$ is coherent, comonotonic additive and non-independent additive, $Q_{C_{\bar{h}}}(\mathbf{w} \mid \mathbf{R})$ satisfies Reverse Risk Degeneracy.

(DI)- Follows the proof of Proposition 1.

(M)- Follows the proof of Proposition 1.

(TI,H)- Since $\bar{h}($.$) is convex, C_{h}(R)$ is translation invariant and homogeneous of degree one. Therefore $\underline{Q}_{C_{h}}(\mathbf{w} \mid \mathbf{R})$ is translation invariant and homogeneous of degree one.

(S)- Suppose that $R_{1}, \ldots, R_{N}$ is exchangeable. It is straightforward to verify that $Q_{C_{h}}(\mathbf{w} \mid \mathbf{R})$ 
is symmetric.

\section{A.3.2 Necessity}

For the converse, suppose that $Q_{C_{h}}(\mathbf{w} \mid \mathbf{R})$ satisfies our axioms and let us show that $\bar{h}($. is convex. To do so, we proceed by contradiction. Suppose that $h($.) is not convex. It is straightforward to verify that $Q_{C_{h}}(\mathbf{w} \mid \mathbf{R})$ is not concave (Wang, Young, and Panjer, 1997).

\section{A.4 Proposition 4}

Follows from Proposition 1.

\section{A.5 Proposition 5}

Follows from Proposition 2.

\section{A.6 Proposition 6}

\section{A.6.I Portfolio variance}

A.6.I.I Sufficiency Suppose that assets have identical variances and let us show that the portfolio variance satisfies our axioms. It is straightforward to verify that if assets have identical variances i.e $\underset{i}{\sigma^{2}}=\underline{\sigma}^{2}$, then

$$
\mathbf{w}^{\mathrm{T}} \boldsymbol{\sigma}^{2}-\sigma^{2}(\mathbf{w} \mid \mathbf{R})=\underline{\sigma}^{2}-\sigma^{2}(\mathbf{w} \mid \mathbf{R})
$$

From (40) and Proposition 1, it follows that $\sigma^{2}(\mathbf{w} \mid \mathbf{R})$ satisfies our axioms.

A.6.1.2 Necessity For the converse, suppose that $\sigma^{2}(\mathbf{w} \mid \mathbf{R})$ our axioms and let us show that assets have identical variances. To do so, we proceed by contradiction. Suppose that asset variances are not identical and without the loss of generality that $N \geq$ such that $\sigma_{1}^{2}<\sigma_{2}^{2}$. Then $\sigma^{2}\left(\boldsymbol{\delta}_{1} \mid \mathbf{R}\right)<\sigma^{2}\left(\boldsymbol{\delta}_{2} \mid \mathbf{R}\right)$. Thus $\sigma^{2}(\mathbf{w} \mid \mathbf{R})$ fails Size Degeneracy. From the failure of Size Degeneracy, it is straightforward to prove that $\left.\sigma^{2}(\mathbf{y}) \mathbf{R}\right)$ also fails Risk Degeneracy and Reverse Risk Degeneracy. This contradicts our hypothesis that $\sigma^{2}(\mathbf{Y} \mathbf{R}$ satisfies our axioms. As a consequence, if $\sigma^{2} \mathbf{w} \mathbf{R}$ satisfies our axioms, then assets have identical variances. 


\section{A.6.2 Diversification ratio}

Because the standard-deviation is convex, positive homogeneous (with $\kappa=1$ ), translation invariant (with $\eta=0$ ) and is reverse lower comonotonic additive, from part (iv) of Proposition 6, $\mathrm{DR}_{\sigma}$ satisfies our axioms. It follows that $\mathrm{DR}(\mathbf{w} \mid \mathbf{R})=\frac{1}{\mathrm{DR}(\mathbf{w} \mid \mathbf{R})_{\delta}}$ also satisfies our axioms.

\section{A.6.3 Embrechts, Furrer, and Kaufmann's (2009) class measures}

See the proof of Proposition 3.

\section{A.6.4 Tasche's (2006) class measures}

(QC)- Since $Q($.$) is convex and \sum_{i=1}^{N} w_{i} Q\left(R_{i}\right)$ is linear on W, from Avriel et al (2010), $\mathrm{DR}_{e}$ (w $\mathbf{R}$ js quasi-concave.

$(\mathrm{SD})-\mathrm{DR}_{e}\left(\boldsymbol{\delta}_{i} \mathbf{R}\right)=\frac{e\left(R^{i}\right)}{e(R)}=1$, for each $i \in \mathbf{I}_{N}$.

(RD)- Since $A_{i}=A, R_{i}=R$ for each $i \in \mathbf{I}_{N}$. Then $\operatorname{DR}_{e}\left(\boldsymbol{\delta}_{i} \mathbf{R}\right)=\frac{e(R)}{e(R)}=\mathbf{1}=\underline{\Phi}$ for each $i_{\in} \mathbf{I} N$. (RRD)- By assumption that $Q_{j}$ is reverse upper comonotonic additive.

(DI)- Follows the proof of Proposition 1.

(M)- Follows the proof of Proposition 1.

(TI)- Since $Q($.$) is translation invariant i.e. Q(R+a)=Q(R)-\eta a$ with $R$ is a random variable,

If $\eta=0$,

$$
\underset{\varepsilon}{\operatorname{DR}\left(\mathbf{w} \mid \mathbf{R}_{\mathrm{A}+}\right)=}{ }_{a} \frac{Q\left(\mathbf{w}^{\mathrm{T}} \mathbf{R}_{\mathrm{A}}\right)-\eta a}{\mathbf{w}^{\mathrm{T}} Q\left(\mathbf{R}_{\mathrm{A}}\right)-\eta a} .
$$

$$
\mathrm{DR}_{e}\left(\mathbf{w} \mid \mathbf{R}_{\mathrm{A}+a}\right)=\mathrm{DR}_{e}\left(\mathbf{w} \mid \mathbf{R}_{\mathrm{A}}\right)
$$


If $\eta \neq 0$,

$$
\begin{aligned}
& \frac{\partial \mathrm{DR}_{e}\left(\mathbf{w} \mid \mathbf{R}_{\mathrm{A}+a}\right)}{\partial a}=\frac{\eta\left(Q\left(\mathbf{w}^{\mathrm{T}} \mathbf{R}_{\mathrm{A}}\right)-\mathbf{w}^{\mathrm{T}} Q\left(\mathbf{R}_{\mathrm{A}}\right)\right)}{\left(\mathbf{w}^{\mathrm{T}} Q(\mathbf{R})-\eta a^{2}\right.} \leq \mathrm{O}, \\
& \text { J }{ }_{a} \lim _{-\infty} \operatorname{DR}_{e}\left(\mathbf{w} \mid \mathbf{R}_{\mathrm{A}+a}\right)=\mathbf{1} \text {, } \\
& { }_{a} \lim _{+\infty} \operatorname{DR}_{e}\left(\mathbf{w} \mid \mathbf{R}_{\mathrm{A}+a}\right)=\mathbf{1},
\end{aligned}
$$

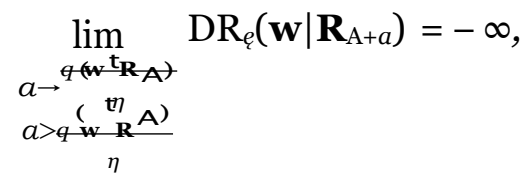

$$
\begin{aligned}
& \lim _{a \rightarrow q\left(\mathbf{w} \mathbf{R}^{A} \mathbf{A}\right)} \operatorname{DR}_{e}\left(\mathbf{w} \mid \mathbf{R}_{\mathrm{A}+a}\right)=\infty . \\
& a<q\left(\begin{array}{c}
\mathbf{w}^{\eta} \mathbf{R} \\
\eta
\end{array}\right)
\end{aligned}
$$

(H)- If $Q($.$) is homogeneous i.e. Q(b R)=b^{\kappa} Q(R)$ with $R$ a random variable,

$$
\begin{aligned}
\operatorname{DR}_{e}(\mathbf{w} \mid b \mathbf{R}) & =\frac{Q\left(b \mathbf{w}^{\mathrm{T}} \mathbf{R}\right)}{\mathbf{w}^{\top} Q(b \mathbf{R})} \\
& =\frac{b^{\kappa} Q\left(\mathbf{w}^{\top} \mathbf{R}\right)}{b^{\kappa} \mathbf{w}^{\mathrm{T}} Q(\mathbf{R})} \\
\mathrm{DR}_{\ell}(\mathbf{w} \mid b \mathbf{R}) & =\mathrm{DR}_{\ell}(\mathbf{w} \mid \mathbf{R})
\end{aligned}
$$

(S)- Follows the proof of Proposition 3.

\section{References}

Abdellaoui, M.: A genuine rank-dependent generalization of the Von Neumann-Morgenstern expected utility theorem. Econometrica 7o, 717-736, (2002)

Aldous, D.J.: Exchangeability and related topics. In: École d'été de probabilités de SaintFlour XIII-1983, pp 1-198. Springer (1985)

Artzner, P., F. Delbaen, J.M. Eber, and D. Heath.: Coherent measures of risk. Mathematical Finance 9, 203-228, (1999)

Aumann, R.J., and Serrano, R.: An economic index of riskiness. Journal of Political Economy 116, 810-836, (2008) 
Avriel, M., W. Diewert, S. Schaible, and I. Zang.: Generalized Concavity, Society for Industrial and Applied Mathematics. Classics in Applied Mathematics. Society for Industrial and Applied Mathematics (SIAM), (2010).

Bank for International Settlements. Basel Committee on Banking Supervision.: Developments in modelling risk aggregation, (2010)

--— .: Consultative document October 2013. Fundamental review of the trading book: A revised market risk framework, (2013)

Barnea, A., and D.E. Logue.: Stock-market based measures of corporate diversification. Journal of Industrial Economics 22, 51-60, (1973)

Bignozzi, V., T. Mao, B. Wang, and R. Wang.: Diversification limit of quantiles under dependence uncertainty. Extremes 19, 143-170, (2016)

Booth, D.G., and E.F. Fama.: Diversification returns and asset contributions. Financial Analysts Journal 48, 26-32, (1992)

Bouchaud, J.P., M. Potters, and J.P. Aguilar.: Missing information and asset allocation. Science \& Finance (CFM) Working paper archive 500045, (1997)

Bouchey, P., V. Nemtchinov, A. Paulsen, and D.M. Stein.: Volatility harvesting: Why does diversifying and rebalancing create portfolio growth? Journal of Wealth Management 15, 26-35, (2012)

Carli, T., R. Deguest, and L. Martellini.: Improved risk reporting with factor-based diversification measures. EDHEC-Risk Institute Publications, (2014)

Carmichael, B., G.B. Koumou, and K. Moran.: Unifying portfolio diversification measures using Rao's quadratic entropy. CRREP Working Paper 2015-02, (2015)

---.: Rao's quadratic entropy and maximum diversification indexation. Quantitative Finance 18, 1017-1031, (2018)

Chambers, D., and J.S. Zdanowicz.: The limitations of diversification return. Journal of Portfolio Management 4o, 65-76, (2014) 
Chateauneuf, A., and G. Lakhnati.: From sure to strong diversification. Economic Theory 32, 511-522, (2007)

Chateauneuf, A., and J.M. Tallon.: Diversification, convex preferences and non-empty core in the Choquet expected utility model. Economic Theory 19, 509-523, (2002)

Chateauneuf, A., and P. Wakker.: An axiomatization of cumulative prospect theory for decision under risk. Journal of Risk and Uncertainty 18, 137-145, (1999)

Chen, O., and T. Hu.: Extreme-aggregation measures in the RDEU model. Statistics \& Probability Letters 148, 155-163, (2019)

Cheung, K.C.: Upper comonotonicity. Insurance: Mathematics and Economics 45, 35-40, (2009)

Chew, S.H., and L.G. Epstein.: A unifying approach to axiomatic non-expected utility theories. Journal of Economic Theory 49, 207-240, (1989)

Chew, S.H., L.L. Epstein, and P.P. Wakker.: A unifying approach to axiomatic non-expected utility theories: Correction and comment. Journal of Economic Theory 59, 183-188, (1993)

Choueifaty, Y., and Y. Coignard.: Toward maximum diversification. Journal of Portfolio Management 35, 40-51, (2008)

Choueifaty, Y., T. Froidure, and J. Reynier.: Properties of the most diversified portfolio. Journal of Investment Strategies 2, 49-70, (2013)

Cohen, M.D.: Risk-aversion concepts in expected- and non-expected-utility models. Geneva Papers on Risk and Insurance Theory 2o, 73-91, (1995)

Committee of European Banking Supervisors.: CEBS's position paper on the recognition of diversification benefits under Pillar 2, (2010)

Committee of European Insurance and Occupational Pensions Supervisors.: CEIOPS' advice for level 2 implementing measures on Solvency II: SCR standard formula article 111(d) correlations, (2010a) 
-_-.: Solvency II calibration paper, (2010b)

Bernard, C. and S. Vanduffel.: Risk aggregation and diversification. Canadian Institute of Actuaries Committee on Risk Management and Capital Requirements Research Paper, (2016).

De Giorgi, E.G. and O. Mahmoud.: Diversification preferences in the theory of choice. Decisions in Economics and Finance 39, 143-174, (2016a)

---.: Naive diversification preferences and their representation. arXiv preprint arXiv:1611.01285, (2016b)

Degen, M., D.D. Lambrigger, and J. Segers.: Risk concentration and diversification: Secondorder properties. Insurance: Mathematics and Economics 46, 541-546, (2010)

Deguest, R., L. Martellini, and A. Meucci.: Risk parity and beyond-from asset allocation to risk allocation decisions. SSRN Working Paper, (2013)

Dekel, E.: Asset demand without the independence axiom. Econometrica 57, 163-169, (1989)

Denuit, M., J. Dhaene, M. Goovaerts, R. Kaas, and R. Laeven.: Risk measurement with equivalent utility principles. Statistics \& Decisions 24, 1-25, (2006)

Denuit, M., J. Dhaene, and M. Van Wouwe.: The economics of insurance: a review and some recent developments. DTEW Research Report 9917, 1-35, (1999)

Dhaene, J., M. Denuit, M.J. Goovaerts, R. Kaas, and D. Vyncke.: The concept of comonotonicity in actuarial science and finance: Applications. Insurance: Mathematics and Economics 31, 133-161, (2002a)

---.: The concept of comonotonicity in actuarial science and finance: Theory. Insurance: Mathematics and Economics 31, 3-33, (2002b)

Dhaene, J., M. Denuit, and S. Vanduffel.: Correlation order, merging and diversification. Insurance: Mathematics and Economics 45, 325-332, (2009) 
Dhaene, J., S. Vanduffel, and M.J. Goovaerts.: Comonotonicity. In: Encyclopedia of Quantitative Risk Assessment and Analysis, John Wiley \& Sons, Ltd (2008).

Dong, J., K.C. Cheung, and H. Yang.: Upper comonotonicity and convex upper bounds for sums of random variables. Insurance: Mathematics and Economics 47, 159-166, (2010)

Embrechts, P., H. Furrer, and R. Kaufmann.: Different kinds of risk. In: Handbook of Financial Time Series, pp 729-751. Springer (2009).

Embrechts, P., G. Puccetti, and L. Rüschendorf. . Model uncertainty and VaR aggregation. Journal of Banking \& Finance 37, 2750-2764, (2013)

Embrechts, P., B. Wang, and R. Wang.: Aggregation-robustness and model uncertainty of regulatory risk measures. Finance and Stochastics 19, 763-790, (2015)

Epstein, L. G., and S.M. Tanny.: Increasing generalized correlation: a definition and some economic consequences. Canadian Journal of Economics 13, 16-34, (1980)

Evans, J.L. and S.H. Archer.: Diversification and the reduction of dispersion: an empirical analysis. Journal of Finance 23, 761-767, (1968)

Fernholz, R.: Diversification. In: Encyclopedia of Quantitative Finance, John Wiley \& Sons, Ltd (2010)

Föllmer, H., and A. Schied.: Convex measures of risk and trading constraints. Finance and Stochastics 6, 429-447, (2002)

Fernholz, R.: Convex Risk Measures. In: Encyclopedia of Quantitative Finance, John Wiley \& Sons, Ltd (2010)

Follmer, H., and S. Weber.: The axiomatic approach to risk measures for capital determination. Annual Review of Financial Economics 7, 301-337, (2015)

Frahm, G., and C. Wiechers.: A diversification measure for portfolios of risky assets. In: Advances in Financial Risk Management, Palgrave Macmillan, pp 312-330. London (2013)

Frittelli, M., and E.R. Gianin.: Putting order in risk measures. Journal of Banking \& Finance 26, 1473-1486, (2002) 
-- -.: Law Invariant Convex Risk Measures. In: Advances in mathematical economics, pp 33-46. Springer Tokyo (2005)

Geissel, S., J. Sass, and F.T. Seifried.: Optimal expected utility risk measures. Statistics \& Risk Modeling 35, 73-87, (2018)

Goetzmann, W.N., and A. Kumar.: Equity portfolio diversification. Review of Finance 12, $433-463,(2008)$

Goovaerts, M.J., R. Kaas, R.J. Laeven, and Q. Tang.: A comonotonic image of independence for additive risk measures. Insurance: Mathematics and Economics 35, 581-594, (2004)

Holton, L.: Is Markowitz wrong? Market turmoil fuels nontraditional approaches to managing investment risk. Journal of Financial Planning 22, 20-26, (2009)

Hua, L., and H. Joe.: Tail comonotonicity: Properties, constructions, and asymptotic additivity of risk measures. Insurance: Mathematics and Economics 51, 492-503, (2012)

Ilmanen, A., and J. Kizer.: The death of diversification has been greatly exaggerated. Journal of Portfolio Management 38, 15-27, (2012)

Koumou, B.G.: Diversification and portfolio theory: A review. Conditionally accepted for publication in Financial Markets and Portfolio Management, (2019)

Lhabitant, F.S.: Portfolio diversification. Elsevier, Saint Louis (2017).

Lintner, J.: The valuation of risk assets and the selection of risky investments in stock portfolios and capital budgets. Review of Economics and Statistics 47, 13-37, (1965)

Maillard, S., T. Roncalli, and J. Teiletche.: The properties of equally weighted risk contribution portfolios. Journal of Portfolio Management 36, 60-70, (2010)

Mao, T., W. Lv, and T. Hu.: Second-order expansions of the risk concentration based on CTE. Insurance: Mathematics and Economics 51, 449-456, (2012)

Markowitz, H.: Portfolio Selection. Journal of Finance 7, 77-91, (1952) 
-—-- . Portfolio Selection: Efficient Diversification of Investments. Malden, Massachusetts: Blackwell Publishers, Inc. Second edition (1959).

Marshall, A.W., I. Olkin, and B.C. Arnold.: Inequalities: Theory of Majorization and its Applications. Springer Series in Stastictics. Second edition (2011).

Meucci, A.: Managing diversification. Risk 22, 74-79, (2009)

Meucci, A., A. Santangelo, and R. Deguest.: Measuring portfolio diversification based on optimized uncorrelated factors, (2014). SSRN Working Paper.

- - .: Risk budgeting and diversification based on optimized uncorrelated factors, (2015). SSRN Working Paper.

Meyer, J.: Two-moment decision models and expected utility maximization. American Economic Review 77, 421-430, (1987)

Miccolis, J.A. and M. Goodman.: Next generation investment risk management: Putting the 'modern' back in modern portfolio theory. Journal of Financial Planning 25, 44-51, (2012)

Müller, A.: Certainty equivalents as risk measures. Brazilian Journal of Probability and Statistics 21, 1-12, (2007)

Nakamura, Y.: Rank dependent utility for arbitrary consequence spaces. Mathematical Social Sciences 29, 103-129, (1995)

--—.: Mean-variance utility. Journal of Economic Theory 160, 536-556, (2015)

Nam, H. S., Q. Tang, and F. Yang.: Characterization of upper comonotonicity via tail convex order. Insurance: Mathematics and Economics 48, 368-373, (2011)

Pedersen, S.C., and E.S. Satchell.: An extended family of financial-risk measures. Geneva Papers on Risk and Insurance Theory 23, 89-117, (1998)

Pratt, J.W.: Risk Aversion in the small and in the large. Econometrica 32, 122-136, (1964) 
Qian, E.: On the financial interpretation of risk contribution: Risk budgets do add up. Journal of Investment Management 4, 41-51, (2006)

- - -.: Diversification return and leveraged portfolios. Journal of Portfolio Management $38,14-25$, (2012)

Quiggin, J.: A theory of anticipated utility. Journal of Economic Behavior \& Organization 3, 323-343, (1982)

Quiggin, J., and P. Wakker.: The axiomatic basis of anticipated utility: A clarification. Journal of Economic Theory 64, 486-499, (1994)

Richard, S.F.: Multivariate risk aversion, utility independence and separable utility functions. Management Science 22, 12-21, (1975)

Rockafellar, R.T., and S. Uryasev.: Optimization of conditional value-at-risk. Journal of Risk 2, 21-41, (2000)

- - .: Conditional value-at-risk for general loss distributions. Journal of Banking \& Finance 26, 1443-1471, (2002)

Rockafellar, R.T., S. Uryasev, and M. Zabarankin.: Generalized deviations in risk analysis. Finance and Stochastics 10, 51-74, (2006)

Roncalli, T.: Introduction to Risk Parity and Budgeting. Chapman \& Hall/CRC Financial Mathematics Series, (2013)

Ross, S.A.: The arbitrage theory of capital asset pricing. Journal of Economic Theory 13, 341-360, (1976)

Rudin, A.M., and J. Morgan.: A portfolio diversification index. Journal of Portfolio Management 32, 81-89, (2006)

Ryan, M.J.: Risk aversion in RDEU. Journal of Mathematical Economics 42, 675-697, (2006)

Salazar Flores, Y.S., R.J. Bianchi, M.E. Drew, and S. Trück.: The diversification delta: A different perspective. Journal of Portfolio Management 43, 112-124, (2017) 
Segal, U.: Anticipated utility: A measure representation approach. Annals of Operations Research 19, 359-373, (1989)

----.: The measure representation: A correction. Journal of Risk and Uncertainty 6, 99-107, (1993a)

-——.: Order indifference and rank-dependent probabilities. Journal of Mathematical Economics 22, 373-397, (1993b)

Sereda, E., E. Bronshtein, S. Rachev, F. Fabozzi, W. Sun, and S. Stoyanov.: Distortion risk measures in portfolio optimization. In: Handbook of Portfolio Construction, (J. Guerard, ed), John B., pp 649-673. Springer US (2010)

Sharpe, W.F.: Capital asset prices: A theory of market equilibrium under conditions of risk. Journal of Finance 19, 425-442, (1964)

--—.: Risk, Market Sensitivity and Diversification. Financial Analysts Journal 28, 74-79, (1972)

Song, Y., and J. Yan. . The representations of two types of functionals on $L^{\infty}$ \&, ampl $L^{\infty} \Omega$, , P . Fciepce in China Series A: Mathematics 49, 1376-1382, (2006)

----.: Risk measures with comonotonic subadditivity or convexity and respecting stochastic orders. Insurance: Mathematics and Economics 45, 459-465, (2009)

Statman, M.: Is Markowitz Wrong? Investment Lessons from the Financial Crisis. Journal of Portfolio Management 40, 8-11, (2013)

Statman, M., and J. Scheid.: Global diversification. Journal of Investment Management 3, 1-11, (2005)

Tasche, D.: Measuring sectoral diversification in an asymptotic multifactor framework. Journal of Credit Risk 2, 33-55, (2006)

Tong, B., C. Wu, and W. Xu.: Risk concentration of aggregated dependent risks: The second-order properties.Insurance: Mathematics and Economics 5o, 139-149, (2012) 
Tsanakas, A., and E. Desli.: Risk measures and theories of choice. British Actuarial Journal 9, 959-991, (2003)

Vermorken, M.A., F.R. Medda, and T. Schroder.: The diversification delta: A highermoment measure for portfolio diversification. Journal of Portfolio Management 39, 67-74, (2012)

Wakker, P.: Separating marginal utility and probabilistic risk aversion. Theory and Decision 36, 1-44, (1994)

Wakker, P., and A. Tversky.: An axiomatization of cumulative prospect theory. Journal of Risk and Uncertainty 7, 147-175, (1993)

Wang, R., V. Bignozzi, and A. Tsanakas.: How superadditive can a risk measure be? SIAM Journal on Financial Mathematics 6, 776-803, (2015)

Wang, S.S., V.R. Young, and H.H. Panjer.: Axiomatic characterization of insurance prices. Insurance: Mathematics and Economics 21, 173-183, (1997)

Wei, L.X., Y. Ma, and Y.J. Hu.: Risk measures with comonotonic subadditivity or convexity on product spaces. Applied Mathematics-A Journal of Chinese Universities 30, 407-417, (2015)

Willenbrock, S.: Diversification return, portfolio rebalancing, and the commodity return puzzle. Financial Analysts Journal 67, 42-49, (2011)

Yaari, M.E.: The dual theory of choice under risk. Econometrica 55, 95-115, (1987)

Yu, J.R., W.Y. Lee, and W.J.P. Chiou.: Diversified portfolios with different entropy measures. Applied Mathematics and Computation 241, 47-63, (2014)

Zhou, R., R. Cai, and G. Tong.: Applications of entropy in finance: A review. Entropy 15, 4909-4931, (2013) 